\title{
Dipolar Excitation of a Perfectly Electrically Conducting Spheroid in a Lossless Medium at the Low-Frequency Regime
}

\author{
Panayiotis Vafeas (iD) \\ Department of Chemical Engineering, University of Patras, 26504 Patras, Greece \\ Correspondence should be addressed to Panayiotis Vafeas; vafeas@chemeng.upatras.gr
}

Received 2 September 2017; Accepted 13 March 2018; Published 26 April 2018

Academic Editor: Antonio Scarfone

Copyright (C) 2018 Panayiotis Vafeas. This is an open access article distributed under the Creative Commons Attribution License, which permits unrestricted use, distribution, and reproduction in any medium, provided the original work is properly cited.

\begin{abstract}
The electromagnetic vector fields, which are scattered off a highly conductive spheroid that is embedded within an otherwise lossless medium, are investigated in this contribution. A time-harmonic magnetic dipolar source, located nearby and operating at low frequencies, serves as the excitation primary field, being arbitrarily orientated in the three-dimensional space. The main idea is to obtain an analytical solution of this scattering problem, using the appropriate system of spheroidal coordinates, such that a possibly fast numerical estimation of the scattered fields could be useful for real data inversion. To this end, incident and scattered as well as total fields are written in a rigorous low-frequency manner in terms of positive integral powers of the realvalued wave number of the exterior environment. Then, the Maxwell-type problem is converted to interconnected Laplace's or Poisson's equations, complemented by the perfectly conducting boundary conditions on the spheroidal object and the necessary radiation behavior at infinity. The static approximation and the three first dynamic contributors are sufficient for the present study, while terms of higher orders are neglected at the low-frequency regime. Henceforth, the 3D scattering boundary value problems are solved incrementally, whereas the determination of the unknown constant coefficients leads either to concrete expressions or to infinite linear algebraic systems, which can be readily solved by implementing standard cut-off techniques. The nonaxisymmetric scattered magnetic and electric fields follow and they are obtained in an analytical compact fashion via infinite series expansions in spheroidal eigenfunctions. In order to demonstrate the efficiency of our analytical approach, the results are degenerated so as to recover the spherical case, which validates this approach.
\end{abstract}

\section{Introduction}

The fundamental principles of classical electromagnetism [1], initially introduced by the pioneer in the field James Clerk Maxwell, represent the basic foundation of the low-frequency scattering theory [2]. Many physical applications that are associated with the response of receivers with arbitrary shape, embedded within various media, when they are excited by several primary sources, still stand in the frontline of scientific research. Indeed, let us give emphasis to some of them like two-phase composites, Earth's subsurface probing for mineral exploration, identification of cavities or other underground detection such as UneXploded Ordnance and buried objects, and scattering by chiral material in either chiral or nonchiral environments. Obviously, the range of the applied frequencies varies according to each case. However, low-frequency interactions exhibit a particular interest for numerous real-life problems that occur in physical areas, which are related to the present contribution. By deciphering the implicated fields to each case, information about main parameters like orientations, sizes, shapes, and magnetic and electric properties of the anomalies brings insight into the field behavior. Nevertheless, this is not at all an easy task, since the situation of an inversion scheme [3] cannot be tackled in a robust-like manner, unless efficient models of the field distribution and strong effective mathematical tools [4] are available, since in practice the measurement and the identification of the scatterer are needed simultaneously.

The large amount of vector data, the electromagnetic and geometrical complexity of the different media involved, the many configurations of sources and receivers, and the uncertainty resulting from datasets containing both the 
contribution of the incident and scattered fields explain the interest of solving forward and inverse electromagnetic scattering problems, first at the modeling stage and later at the inversion stage. Therefore, the already ample library of scattering by simple shapes using analytical methods is open to accept new and useful semianalytical and, why not, analytical results. In the last decade, a lot of articles were published in that direction and many references are available in the literature. Collectively, we pertain to the low-frequency scattering by a perfectly electrically conducting sphere in a conductive environment, illuminated by an arbitrarily orientated and settled magnetic dipole source field [5]. There, the 3D scattered electromagnetic fields have assumed a complete analytical formalism in terms of closed-type solutions, while a numerical implementation has validated almost perfectly the results via a comparison with the exact Mie series expansion [6]. However, this work was a first introduction to scattering by metallic objects that reflect the complete isotropy of the three-dimensional space. Moving to more complicated geometries for the same physical consideration such as spheroids [7] or ellipsoids [8], one can realize that the orientation of the body, save the position or the size, plays a crucial role not only to the mathematical treatment but also to the computational elaboration of the corresponding fields. Moreover, it is obvious that the more general the shape becomes, the more difficulty is added to a possible inversion, which, by the way, is the final goal when we deal with real-life applications. It is to this end that the utilization of elaborate numerical techniques is inevitable, as it is demonstrated in [9] for the identification of an orebody of approximately infinite conductivity, which is buried in Earth, by using an equivalent perfectly conducting triaxial model ellipsoid. High-contrast cases, where the target has an extremely large conductivity with respect to the surrounding host environment and hence is considered to be impenetrable, motivated researchers to embark upon much more complex patterns, such as the two-sphere case for two closely adjacent metallic objects [10] or the circumstance of a nonpenetrable, that is, perfect conductor, ring torus [11], both articles presuming a conductive occupied medium. These reports, each one containing a sufficient reference list, are a small but representative sample of displaying the inconvenience induced in performing analytical techniques when incorporating with different geometrical models [12, 13]. As a matter of fact, the difficulty increases due to the emergence of intricate eigenfunctions [14, 15], dealing with the involved potentials.

This project is focused now on the adjustment of the lowfrequency diffusive scattering theory in solving the particular problem of identifying impenetrable metallic bodies in a lossless, that is, perfect dielectric, medium, aiming to follow the similar successful path of other contributions to the field. Bearing in mind the few, but with quite good results, attempts of simulating the environment as lossless and not conductive (e.g., see [16] for the case of two metallic spheres almost touching and [17] for the toroidal case in this direction), we realize the necessity of dealing with such brute-force analytical models. Seeking for different body structures in order to attain the representation of all kind of anomalies in lossless media, as much effectively as possible, the present paper deals with the spheroidal configuration. Although research on quasi-static fields around spheroids is vast, there are still elementary properties to be discovered; thus the demand for comparable investigations stays on continuously even nowadays. Consequently, here we extend the research to a perfectly reflective spheroid, embedded in a lossless ambient, where the appropriate and best fitting system for the particular modeling purposes is the spheroidal coordinate system $[12,13]$. The prolate spheroidal geometry is considered, since the oblate one is readily obtained via a simple analytical transformation, while limiting cases are also available. On the other hand, a time-harmonic magnetic dipole source, acting as the primary source, operates at the low-frequency regime and generates the three-dimensional incident electromagnetic waves, which propagate towards the scatterer. Then, the spheroidal-shaped body responds to the excitation and generates the scattered magnetic and electric fields, where their summation comprises the total fields.

The physical nature of the problem urges us to cope with the following low-frequency technique, where the incident, the scattered, and the total electromagnetic fields are expanded in terms of positive integral powers of $(i k)$, where $k$ denotes the real wave number of the exterior medium at the operation frequency. Here, we elaborate the 3D vector fields at each order of $(i k)^{n}$ for every $n \in \mathbb{N}$, whereas our problem is transformed into a sequence of coupled boundary value scattering problems. The model is mathematically formulated in terms of second-order Laplace's and Poisson's partial differential equations, accompanied by the appropriate perfectly electrically conducting boundary conditions, that is, cancellation of normal magnetic and tangential electric fields, reflecting the impenetrable character of the boundary of the spheroid-type metallic targets. The Silver-Müller radiation conditions at infinity are applied as well. We restrict our analysis to the important terms of the expansions, confined by the static Rayleigh term for $n=0$ and the dynamic terms for $n=1,2,3$, since terms of higher orders, that is, $n \geq 4$, are small contributors due to the low frequencies of which the source operates and, consequently, they are neglected. The analytical procedure sketched above leads either to closed relationships or to infinite linear algebraic systems for the determination of the unknown constant coefficients of the implied potential functions, where the latter can be solved approximately by enforcing usual cut-off methods. The threedimensional scattered magnetic and electric fields for each $n=0,1,2,3$ admit closed-type forms via infinite series expansions in view of spheroidal eigenfunctions $[14,15]$. The key to the validation of our approach is the degeneration of the obtained formulae in order to recover the spherical case.

The rest of the paper itself is organized as follows. In Section 2, a detailed physical interpretation and the theoretical basis via an analytical mathematical formulation are sketched, which incorporate the general features, independently of any geometry. Thereafter, a brief configuration of the prolate spheroidal system and the eigenfunctions associated with it is developed in Section 3, whereas the sought solution is constructed step by step and the electromagnetic lowfrequency fields assume infinite series expansions in terms 
of prolate spheroidal eigenfunctions. Though the transformation of these geometrical and analytical attributes to the oblate spheroidal case is presented, some interesting limiting occasions are also discussed. Section 4 is devoted to the validation of the aforementioned analytical method via a proper reduction to the corresponding spherical problem that illustrates the above results. Finally, a short conclusion and discussion, containing an outline of our work and future steps, follow in Section 5, while an updated reference list is displayed immediately after.

\section{Physical and Mathematical Interpretation}

Suppose a solid spheroidal body, either prolate or oblate, which by physical means has an approximate conductivity $\sigma_{b} \rightarrow+\infty$ with respect to the surrounding homogeneous, isotropic, and nonmagnetic lossless medium of conductivity $\sigma \cong 0$. The constructed body-medium system is assumed to occupy the three-dimensional space $V\left(\mathbb{R}^{3}\right)$, while the interface that distinguishes the two regions is actually the smooth surface $S$ of the perfectly electrically conducting object. Hence, the area of electromagnetic scattering is spread to the unbounded domain of dielectric permittivity $\varepsilon$ and magnetic permeability $\mu$, approximated by the corresponding one $\mu_{0}$ of the vacuum, outside the spheroid, yielding exteriortype problems. By definition of the given low circular frequency $\omega$, the real-valued wave number of the medium is

$$
k=\omega \sqrt{\varepsilon \mu},
$$

where (1) secures the conjecture of low frequencies. Harmonic time-dependence is implied for the fields via multiplication of their spatial part by $\exp (-i \omega t)$ (imaginary unit $i=\sqrt{-1})$; consequently they admit expressions as a function of $\mathbf{r}=x_{1} \widehat{\mathbf{x}}_{1}+x_{2} \widehat{\mathbf{x}}_{2}+x_{3} \widehat{\mathbf{x}}_{3}$, which is expressed via the Cartesian basis $\widehat{\mathbf{x}}_{\kappa}, \kappa=1,2,3$ in Cartesian coordinates $\left(x_{1}, x_{2}, x_{3}\right)$, where this dependence will be omitted henceforth for reasons of writing convenience.

The impenetrable spheroid is excited by a primary vector source field, for example, a magnetic dipole $\mathbf{m}$ for the purposes of this project, having an arbitrary orientation that yields

$$
\mathbf{m}=\sum_{j=1}^{3} m_{j} \widehat{\mathbf{x}}_{j}
$$

being located at $\mathbf{r}=\mathbf{r}_{0}$. The fixed singular point $\mathbf{r}_{0}$ is excluded from the domain $V\left(\mathbb{R}^{3}\right)$ of scattering, since the region of interest is at a reasonably long distance from the source. Thus, we adopt the explicit definition $\Omega \equiv V\left(\mathbb{R}^{3}\right)-\left\{\mathbf{r}_{0}\right\}$ as for the area of electromagnetic activity. According to the simple notation $\mathbf{R}=\mathbf{r}-\mathbf{r}_{0}$ and $R=\left|\mathbf{r}-\mathbf{r}_{0}\right|$, the magnetic dipolar source radiates the primary fields [2]

$$
\begin{aligned}
& \mathbf{H}^{p}\left(\mathbf{r} ; \mathbf{r}_{0}\right)=\frac{1}{4 \pi}\left[\left(k^{2}+\frac{i k}{R}-\frac{1}{R^{2}}\right) \mathbf{m}\right. \\
& \left.\quad-\left(k^{2}+\frac{3 i k}{R}-\frac{3}{R^{2}}\right) \frac{\mathbf{R} \otimes \mathbf{R} \cdot \mathbf{m}}{R^{2}}\right] \frac{e^{i k R}}{R} \quad \text { for } \mathbf{r} \in \Omega,
\end{aligned}
$$

$$
\mathbf{E}^{p}\left(\mathbf{r} ; \mathbf{r}_{0}\right)=\left[\frac{\omega \mu k}{4 \pi}\left(1+\frac{i}{k R}\right) \frac{\mathbf{m} \times \mathbf{R}}{R}\right] \frac{e^{i k R}}{R}
$$

for $\mathbf{r} \in \Omega$,

where the symbol “ $\otimes$ " denotes dyadic product. In terms of Maclaurin's series expansions of the exponent $e^{i k R}$ and implying (1), we perform some extended algebraic calculations by collecting terms as integral powers of $(i k)$ and we retain the adequate number of them that correspond to the static Rayleigh approximation and to the first three dynamic factors. Therefore, the incident magnetic field (3) is rewritten as

$$
\begin{aligned}
& \mathbf{H}^{p}\left(\mathbf{r} ; \mathbf{r}_{0}\right) \\
& =\left[\mathbf{H}_{0}^{p}\left(\mathbf{r} ; \mathbf{r}_{0}\right)+\frac{\mathbf{H}_{2}^{p}\left(\mathbf{r} ; \mathbf{r}_{0}\right)}{2}(i k)^{2}+\frac{\mathbf{H}_{3}^{p}\left(\mathbf{r} ; \mathbf{r}_{0}\right)}{6}(i k)^{3}\right] \\
& \quad+\bigcirc\left((i k)^{4}\right) \quad \text { for } \mathbf{r} \in \Omega,
\end{aligned}
$$

while the corresponding primary incident electric field (4) renders

$$
\begin{aligned}
\mathbf{E}^{p}\left(\mathbf{r} ; \mathbf{r}_{0}\right)= & {\left[\mathbf{E}_{1}^{p}\left(\mathbf{r} ; \mathbf{r}_{0}\right)(i k)+\frac{\mathbf{E}_{3}^{p}\left(\mathbf{r} ; \mathbf{r}_{0}\right)}{6}(i k)^{3}\right] } \\
& +\bigcirc\left((i k)^{4}\right) \quad \text { for } \mathbf{r} \in \Omega,
\end{aligned}
$$

where the input of the fields of order $n \geq 4$ is minor, since $\omega$ and consequently $k$ are very small. On the other hand, the surviving electromagnetic fields for all $n=0,1,2,3$ enjoy

$$
\begin{array}{r}
\mathbf{H}_{0}^{p}\left(\mathbf{r} ; \mathbf{r}_{0}\right)=\frac{\mathbf{m}}{4 \pi} \cdot\left(\frac{3 \mathbf{R} \otimes \mathbf{R}}{R^{2}}-\tilde{\mathbf{I}}\right) \frac{1}{R^{3}}=\frac{\mathbf{m}}{4 \pi} \cdot\left(\nabla \otimes \nabla \frac{1}{R}\right) \\
\text { for } \mathbf{r} \in \Omega,
\end{array}
$$

$$
\begin{aligned}
\mathbf{H}_{2}^{p}\left(\mathbf{r} ; \mathbf{r}_{0}\right) & =-\frac{\mathbf{m}}{4 \pi} \cdot\left(\frac{\mathbf{R} \otimes \mathbf{R}}{R^{2}}+\widetilde{\mathbf{I}}\right) \frac{1}{R} \\
& =\frac{\mathbf{m}}{4 \pi} \cdot\left(\nabla \frac{1}{R} \otimes \mathbf{R}-\frac{\widetilde{\mathbf{I}}}{R}\right) \quad \text { for } \mathbf{r} \in \Omega, \\
\mathbf{H}_{3}^{p}\left(\mathbf{r} ; \mathbf{r}_{0}\right) & =\frac{\mathbf{m}}{4 \pi} \cdot(-4 \widetilde{\mathbf{I}}) \quad \text { for } \mathbf{r} \in \Omega,
\end{aligned}
$$

as far as the magnetic components are concerned, while

$$
\mathbf{E}_{1}^{p}\left(\mathbf{r} ; \mathbf{r}_{0}\right)=\frac{\mathbf{m}}{4 \pi} \sqrt{\frac{\mu}{\varepsilon}} \times \frac{\mathbf{R}}{R^{3}}=-\frac{\mathbf{m}}{4 \pi} \sqrt{\frac{\mu}{\varepsilon}} \times \nabla \frac{1}{R}
$$

for $\mathbf{r} \in \Omega$,

$$
\mathbf{E}_{3}^{p}\left(\mathbf{r} ; \mathbf{r}_{0}\right)=-\frac{\mathbf{m}}{4 \pi} \sqrt{\frac{\mu}{\varepsilon}} \times \frac{3 \mathbf{R}}{R} \quad \text { for } \mathbf{r} \in \Omega,
$$

obtained for the electric elements, where $\mathbf{H}_{1}^{p}=\mathbf{E}_{0}^{p}=\mathbf{E}_{2}^{p}=$ $\mathbf{0}$, as it is indicated by the performed analysis. Expressions 
(7)-(11) are provided in view of the unit dyadic $\widetilde{\mathbf{I}}=\sum_{j=1}^{3} \widehat{\mathbf{x}}_{j} \otimes$ $\widehat{\mathbf{x}}_{j}$, while the handy forms, depicted on the right-hand side of (7), (8), and (10), are based on straightforward computations with the aid of trivial differential identities and by virtue of

$$
\nabla_{\mathbf{r}} \frac{1}{R} \equiv \nabla \frac{1}{R}=-\frac{\mathbf{R}}{R^{3}}=-\nabla_{\mathbf{r}_{0}} \frac{1}{R} \quad \text { for every } \mathbf{r} \in \Omega,
$$

given that $\nabla \otimes \mathbf{r}=\widetilde{\mathbf{I}}$. They contain the fundamental quantity, captured under the action of the gradient operator $\nabla \equiv$ $\nabla_{\mathbf{r}}=\sum_{j=1}^{3} \widehat{\mathbf{x}}_{j}\left(\partial / \partial x_{j}\right)$ (note that the Laplacian is $\Delta \equiv \Delta_{\mathbf{r}}=$ $\left.\sum_{j=1}^{3}\left(\partial^{2} / \partial x_{j}^{2}\right)=\nabla \cdot \nabla\right)$. Though these operators could also be applied on $\mathbf{r}_{0}$, however for reasons of technical clarity we distinguish this feature by introducing the above notation; otherwise it will be instantly designated. In the aftermath, the primary incident fields are perturbed by the solid object, producing the corresponding scattered fields $\mathbf{H}^{s}$ and $\mathbf{E}^{s}$, while the total fields emerge as the summation

$$
\begin{aligned}
\mathbf{H}^{t}(\mathbf{r}) & =\mathbf{H}^{p}\left(\mathbf{r} ; \mathbf{r}_{0}\right)+\mathbf{H}^{s}(\mathbf{r}), \\
\mathbf{E}^{t}(\mathbf{r}) & =\mathbf{E}^{p}\left(\mathbf{r} ; \mathbf{r}_{0}\right)+\mathbf{E}^{s}(\mathbf{r}),
\end{aligned}
$$

for $\mathbf{r} \in \Omega$,

where, since the spheroidal metal body is nonpenetrable, the wave propagation is not possible inside it. By definition of the simple notation $x=p, s, t$, corresponding to primary $(p)$, scattered $(s)$, and total $(t)$ electromagnetic fields, all pairs $\left(\mathbf{H}^{x}, \mathbf{E}^{x}\right)$ satisfy the well-known Maxwell's equations [2]. Yet, as for the scattering components that interest us in the sought solution within $\Omega$, they become

$$
\begin{aligned}
& \nabla \times \mathbf{E}^{\mathcal{s}}(\mathbf{r})=i \omega \mu \mathbf{H}^{\mathcal{s}}(\mathbf{r}) \\
& \nabla \times \mathbf{H}^{\mathcal{s}}(\mathbf{r})=(-i \omega \mathcal{E}+\sigma) \mathbf{E}^{\mathcal{s}}(\mathbf{r}) \stackrel{\sigma \rightarrow 0}{\cong}-i \omega \varepsilon \mathbf{E}^{\mathcal{s}}(\mathbf{r}),
\end{aligned}
$$

which represent a deduced form for our particular circumstance and they are divergence-free; that is,

$$
\nabla \cdot \mathbf{H}^{s}(\mathbf{r})=\nabla \cdot \mathbf{E}^{\mathcal{s}}(\mathbf{r})=0 \quad \text { for } \mathbf{r} \in \Omega,
$$

implying their solenoidal attribute, such that (14) is readily sufficed.

The low-frequency interaction high-contrast cases, as dictated by the physics that we address here, are inherited to the scattered fields and they are postulated by the series

$$
\begin{aligned}
& \mathbf{H}^{s}(\mathbf{r})=\sum_{n=0}^{+\infty} \mathbf{H}_{n}^{s}(\mathbf{r}) \frac{(i k)^{n}}{n !} \\
& \mathbf{E}^{s}(\mathbf{r})=\sum_{n=0}^{+\infty} \mathbf{E}_{n}^{s}(\mathbf{r}) \frac{(i k)^{n}}{n !}
\end{aligned}
$$

where their written fashion is referenced in [2]. Substituting expansions (16) into Maxwell's equations (14), accompanied by (15), we retrieve

$$
\begin{aligned}
& \nabla \times \mathbf{E}_{n}^{s}(\mathbf{r})=n \sqrt{\frac{\mu}{\varepsilon}} \mathbf{H}_{n-1}^{s}(\mathbf{r}), \\
& \nabla \times \mathbf{H}_{n}^{s}(\mathbf{r})=-n \sqrt{\frac{\bar{\varepsilon}}{\mu}} \mathbf{E}_{n-1}^{s}(\mathbf{r}), \\
& \text { with } n \geq 0 \text {, for } \mathbf{r} \in \Omega \text {, } \\
& \nabla \cdot \mathbf{H}_{n}^{s}(\mathbf{r})=0, \quad \nabla \cdot \mathbf{E}_{n}^{s}(\mathbf{r})=0, \\
& \text { with } n \geq 0 \text {, for } \mathbf{r} \in \Omega \text {, }
\end{aligned}
$$

which stand for the low-frequency equivalent expressions. However, for all the reasons described earlier, the behavior of the incident fields (5) and (6) with (7)-(11) reflects exactly the very same physical and mathematical treatment to the scattered fields (16) with (17) and (18); that is, we hold the corresponding nonvanishing terms, attaining fields up to the third order, since the rest of them consist of a minor correction. As a consequence, the scattered magnetic field reads

$$
\begin{aligned}
\mathbf{H}^{s}(\mathbf{r})= & {\left[\mathbf{H}_{0}^{s}(\mathbf{r})+\frac{\mathbf{H}_{2}^{s}(\mathbf{r})}{2}(i k)^{2}+\frac{\mathbf{H}_{3}^{s}(\mathbf{r})}{6}(i k)^{3}\right] } \\
& +\bigcirc\left((i k)^{4}\right) \quad \text { for } \mathbf{r} \in \Omega,
\end{aligned}
$$

while scattered electric field is assumed to be

$$
\mathbf{E}^{s}(\mathbf{r})=\left[\mathbf{E}_{1}^{s}(\mathbf{r})(i k)+\frac{\mathbf{E}_{3}^{s}(\mathbf{r})}{6}(i k)^{3}\right]+\bigcirc\left((i k)^{4}\right)
$$

for $\mathbf{r} \in \Omega$,

considering $\mathbf{H}_{1}^{\mathcal{s}}=\mathbf{E}_{0}^{\mathcal{s}}=\mathbf{E}_{2}^{\mathcal{s}}=\mathbf{0}$, in the absence of the relative incident components. Obviously, taking this into account, the fundamental relations (17) and (18) are immediately fulfilled by the surviving low-frequency fields $\mathbf{H}_{0}^{s}, \mathbf{H}_{2}^{s}, \mathbf{H}_{3}^{s}, \mathbf{E}_{1}^{s}$, and $\mathbf{E}_{3}^{\mathcal{S}}$ arising from (19) and (20) for $n=0,1,2,3$, where their evaluation specifies our final goal. Aiming to understand the contribution of each low-frequency term within (19) and (20), we reinforce (1) and we separate the real from the imaginary part, concluding to

$$
\begin{aligned}
\mathbf{H}^{s}(\mathbf{r})= & {\left[\mathbf{H}_{0}^{s}(\mathbf{r})-\frac{(\omega \sqrt{\varepsilon \mu})^{2}}{2} \mathbf{H}_{2}^{s}(\mathbf{r})\right] } \\
& +\left[-\frac{(\omega \sqrt{\varepsilon \mu})^{3}}{6} \mathbf{H}_{3}^{s}(\mathbf{r})\right] i+\bigcirc\left((i k)^{4}\right)
\end{aligned}
$$

for $\mathbf{r} \in \Omega$,

$$
\begin{aligned}
\mathbf{E}^{s}(\mathbf{r})= & \omega \sqrt{\varepsilon \mu}\left[\mathbf{E}_{1}^{s}(\mathbf{r})-\frac{(\omega \sqrt{\varepsilon \mu})^{2}}{6} \mathbf{E}_{3}^{s}(\mathbf{r})\right] i \\
& +\bigcirc\left((i k)^{4}\right) \quad \text { for } \mathbf{r} \in \Omega,
\end{aligned}
$$


the electric field being purely imaginary, while the magnetic field that is practically measured is a complex-valued function.

In order to proceed to the solution, we are obliged to complete the aforementioned analysis by the exposition of the related to the problem boundary and limiting conditions. Under this aspect, the properly fitted perfectly electrically conducting boundary conditions on the surface $S$ of the spheroidal target are provided in terms of the corresponding outward unit normal vector $\widehat{\mathbf{n}}$ and involve the total fields via

$$
\widehat{\mathbf{n}} \cdot \mathbf{H}^{t}(\mathbf{r})=0, \quad \widehat{\mathbf{n}} \times \mathbf{E}^{t}(\mathbf{r})=\mathbf{0}, \quad \text { for every } \mathbf{r} \in S,
$$

which demand cancellation of the normal component of the total magnetic field and of the tangential component of the total electric field. The low-frequency analogy of (23) turns out to be a combination of it with (16), whereas by virtue of (13) we are led to

$$
\begin{array}{ll}
\widehat{\mathbf{n}} \cdot\left[\mathbf{H}_{n}^{p}\left(\mathbf{r} ; \mathbf{r}_{0}\right)+\mathbf{H}_{n}^{s}(\mathbf{r})\right]=0 & \text { for } n=0,2,3, \\
\widehat{\mathbf{n}} \times\left[\mathbf{E}_{n}^{p}\left(\mathbf{r} ; \mathbf{r}_{0}\right)+\mathbf{E}_{n}^{s}(\mathbf{r})\right]=\mathbf{0} & \text { for } n=1,3 \text { on } S,
\end{array}
$$

while relations (24) refer only to the remaining electromagnetic terms, since $\mathbf{H}_{1}^{x}=\mathbf{E}_{0}^{x}=\mathbf{E}_{2}^{x}=\mathbf{0}$ for any $x=p, s, t$. Additionally, for the implicated functions, the Silver-Müller radiation conditions at infinity for the scattered fields, those being

$$
\lim _{|\mathbf{r}| \rightarrow+\infty}\left[\mathbf{r} \times \nabla \times\left(\begin{array}{c}
\mathbf{H}^{s}(\mathbf{r}) \\
\mathbf{E}^{\mathcal{S}}(\mathbf{r})
\end{array}\right)+i k|\mathbf{r}|\left(\begin{array}{c}
\mathbf{H}^{s}(\mathbf{r}) \\
\mathbf{E}^{\mathcal{S}}(\mathbf{r})
\end{array}\right)\right]=\mathbf{0}
$$

for every $\mathbf{r} \in \Omega$,

must be instantly satisfied, also for the low-frequency regime through (16), giving

$$
\lim _{|\mathbf{r}| \rightarrow+\infty}\left[\mathbf{r} \times \nabla \times\left(\begin{array}{c}
\mathbf{H}_{n}^{s}(\mathbf{r}) \\
\mathbf{E}_{n}^{s}(\mathbf{r})
\end{array}\right)+n|\mathbf{r}|\left(\begin{array}{c}
\mathbf{H}_{n-1}^{s}(\mathbf{r}) \\
\mathbf{E}_{n-1}^{s}(\mathbf{r})
\end{array}\right)\right]=\mathbf{0}
$$

with $n=1,2,3$ for every $\mathbf{r} \in \Omega$,

since, for $n=0$, it holds that $\mathbf{E}_{0}^{\mathcal{S}}=\mathbf{0}$ and $\lim _{|\mathbf{r}| \rightarrow+\infty}(\mathbf{r} \times \nabla \times$ $\mathbf{H}_{0}^{s}$ ) $=\mathbf{0}$ as it is verified from (17). Exterior-type solutions, like in our case, satisfy (25) automatically, as a consequence of the appropriate construction of the potentials via the corresponding eigenfunctions $[14,15]$.

During our analysis, it is supposed that the conductivity of the spheroid approaches infinity. Nevertheless, herein we should include a small discussion about the transition zone, that is, how to treat materials with large, or even comparable with respect to the exterior medium, but not infinite conductivity. In this case, the problem becomes slightly more complicated, since the body becomes penetrable; hence the interior electromagnetic fields exist and contribute to the final solution via the transmission conditions, which are applied on the material's surface instead of (23); see [2] for more details. There, it is shown that conductive or even dielectric particles can resonate with an incident electromagnetic wave over a wide range of modes related to surface excitation. This is due to the excess surface charge that causes the particles of arbitrary composition obtaining metallic properties, a fact especially important for spheres and spheroids. However, this is a completely different situation from the present investigation, concerning applications of importance in other physical areas, for example, in optics.

In summary, we have to manipulate a little bit further the partial differential equations (17) with (18) to obtain easy-to-handle elliptic equations with partial derivatives and, then, use the spheroidal coordinate geometry and analysis, whereby the conditions (24) and (26) are applied, to reach the final desired solution.

\section{Spheroidal Low-Frequency Electromagnetic Fields}

We intend to derive closed analytical formulae for the main nonvanishing scattered electromagnetic fields $\mathbf{H}_{0}^{s}, \mathbf{H}_{2}^{s}, \mathbf{H}_{3}^{s}, \mathbf{E}_{1}^{s}$, and $\mathbf{E}_{3}^{s}$, since $\mathbf{H}_{1}^{s}=\mathbf{E}_{0}^{s}=\mathbf{E}_{2}^{s}=\mathbf{0}$. To achieve that, we have to recover from (17), provided (18), known partial differential equations. To this direction, we apply curl on both sides of Maxwell's equations (17) and we utilize repeatedly the very useful identity $\nabla \times \nabla \times \mathbf{f}=\nabla(\nabla \cdot \mathbf{f})-\Delta \mathbf{f}$ with $\mathbf{f}$ being any vector, where, along with (18), we arrive at a set of boundary value problems, which are coupled to one another, from the static one at $n=0$ to all the rest of the dynamic ones at higher orders up to $n=3$. Those are written explicitly as functions of the scalar $\Phi_{0}^{s}, \Phi_{3}^{s}$ and the vector $\mathbf{X}_{2}^{s}, \mathbf{X}_{3}^{s}$ harmonic potentials,

$$
\begin{aligned}
& \Delta \Phi_{0}^{s}(\mathbf{r})=\Delta \Phi_{3}^{s}(\mathbf{r})=0, \\
& \Delta \mathbf{X}_{2}^{s}(\mathbf{r})=\Delta \mathbf{X}_{3}^{s}(\mathbf{r})=\mathbf{0},
\end{aligned}
$$

for every $\mathbf{r} \in \Omega$,

providing independently

$$
\begin{aligned}
& \Delta \mathbf{H}_{0}^{s}(\mathbf{r})=\mathbf{0} \Longrightarrow \mathbf{H}_{0}^{s}(\mathbf{r})=\nabla \Phi_{0}^{s}(\mathbf{r}), \\
& \text { since } \nabla \cdot \mathbf{H}_{0}^{s}(\mathbf{r})=0, \nabla \times \mathbf{H}_{0}^{s}(\mathbf{r})=\mathbf{0} \text { for } \mathbf{r} \in \Omega,
\end{aligned}
$$

while utilizing (28); then the right-hand side of what follows is known; that is,

$$
\begin{aligned}
\Delta \mathbf{H}_{2}^{s}(\mathbf{r})=2 \mathbf{H}_{0}^{s}(\mathbf{r}) \Longrightarrow & \mathbf{H}_{2}^{s}(\mathbf{r})=\mathbf{X}_{2}^{s}(\mathbf{r})+\mathbf{r} \Phi_{0}^{s}(\mathbf{r}) \\
& \text { with } \nabla \cdot \mathbf{H}_{2}^{s}(\mathbf{r})=0 \text { for } \mathbf{r} \in \Omega,
\end{aligned}
$$

from which we may recover

$$
\mathbf{E}_{1}^{s}(\mathbf{r})=-\frac{1}{2} \sqrt{\frac{\mu}{\varepsilon}} \nabla \times \mathbf{H}_{2}^{s}(\mathbf{r}) \quad \text { for } \mathbf{r} \in \Omega
$$

which serves as the nonhomogeneous part of the equation that follows; that is,

$$
\begin{aligned}
& \Delta \mathbf{E}_{3}^{s}(\mathbf{r})=6 \mathbf{E}_{1}^{s}(\mathbf{r}) \Longrightarrow \\
& \mathbf{E}_{3}^{s}(\mathbf{r})=\mathbf{X}_{3}^{s}(\mathbf{r})+6\left[-\frac{1}{4 \pi} \iiint_{\Omega} \frac{\mathbf{E}_{1}^{s}\left(\mathbf{r}^{\prime}\right)}{\left|\mathbf{r}-\mathbf{r}^{\prime}\right|} d \Omega^{\prime}\right], \\
& \text { with } \nabla \cdot \mathbf{E}_{3}^{s}(\mathbf{r})=0, \quad \mathbf{r} \in \Omega .
\end{aligned}
$$


Moreover, we work similarly to the zeroth-order field to obtain

$$
\begin{aligned}
& \Delta \mathbf{H}_{3}^{s}(\mathbf{r})=\mathbf{0} \Longrightarrow \mathbf{H}_{3}^{s}(\mathbf{r})=\nabla \Phi_{3}^{s}(\mathbf{r}), \\
& \text { since } \nabla \cdot \mathbf{H}_{3}^{s}(\mathbf{r})=0, \nabla \times \mathbf{H}_{3}^{s}(\mathbf{r})=\mathbf{0} \text { for } \mathbf{r} \in \Omega,
\end{aligned}
$$

where we recall that the scattered fields $\mathbf{H}_{0}^{s}, \mathbf{H}_{2}^{s}, \mathbf{H}_{3}^{s}, \mathbf{E}_{1}^{s}$, and $\mathbf{E}_{3}^{s}$ must be determined in the confined scattering region of wave propagation $\Omega \equiv V\left(\mathbb{R}^{3}\right)-\left\{\mathbf{r}_{0}\right\}$. Some extended analysis reveals that, for $n=0$, which corresponds to the Rayleigh approximation term, an uncoupled Laplace's equation is solved for the magnetic field, while there exists no electric field, since the corresponding term for the incident electric field is absent. On the contrary, the dynamic term for $n=1$ reveals the existence of a vector harmonic electric field with a zero-term magnetic field. As for the $n=2$ case, it involves a Poisson's equation for the magnetic field, coupled to the static term. Exploiting appropriately the $n=$ 0 problem, we solve Poisson's equation by introducing a special form of a particular solution, which is actually the outcome of the application of a standard vector identity, using the fact that $\Delta \mathbf{r}=\mathbf{0}$ and $\nabla \otimes \mathbf{r}=\widetilde{\mathbf{I}}$. The incident electric field does not exist, inheriting the same attribute to the scattered one. Finally, at $n=3$ both magnetic and electric fields appear. As far as the magnetic field is concerned, we simply solve an uncoupled Laplace's equation. However, this is not the case for the problem corresponding to the electric field for which the solution is introduced in the form of an integral representation in terms of the fundamental solution of Laplace's equation [14]. Problems (28)-(32) with (27) are supplemented by the perfectly reflecting boundary conditions (24) and the proper limiting behavior (26). It is obvious that the low-frequency magnetic terms of order $n \geq 0$ vary like $1 / R^{3-n}$, while the corresponding electric ones vary like $1 / R^{4-n}$ as $R>0$ increases to infinity. Thus, implying $n=1$ to obtain $\mathbf{E}_{1}^{s}$, integral within (31) is of order $1 / R$, which converges as $R \rightarrow+\infty$.

\subsection{The Prolate Spheroidal Coordinate System with Geometry} and Analysis. For the sake of completeness, we provide a brief analysis of the spheroidal case and especially the prolate spheroidal geometry, since the oblate one can be readily recovered as it will be seen afterwards. Given a fixed positive number $c>0$, which we consider to be the semifocal distance of our system, we define the transformed prolate spheroidal coordinates $(\tau, \zeta, \varphi)$ for every $1 \leq \tau \equiv \cosh \eta<+\infty,-1 \leq \zeta \equiv$ $\cos \theta \leq 1$, and $0 \leq \varphi<2 \pi$ (see Figure 1 ) as

$$
\begin{aligned}
& x_{1}=c \tau \zeta, \\
& x_{2}=c \sqrt{\tau^{2}-1} \sqrt{1-\zeta^{2}} \cos \varphi, \\
& x_{3}=c \sqrt{\tau^{2}-1} \sqrt{1-\zeta^{2}} \sin \varphi
\end{aligned}
$$

with position vector $\mathbf{r}=\left(x_{1}, x_{2}, x_{3}\right)$, where $\mathbf{r}_{0}=\left(x_{10}, x_{20}\right.$, $x_{30}$ ) designate the prescribed source's location. In the limit as $\tau \rightarrow 1^{+}$the prolate spheroid degenerates to the standard focal segment $L_{0}=\{(t, 0,0): t \in[-c, c], c>0\}$, while as

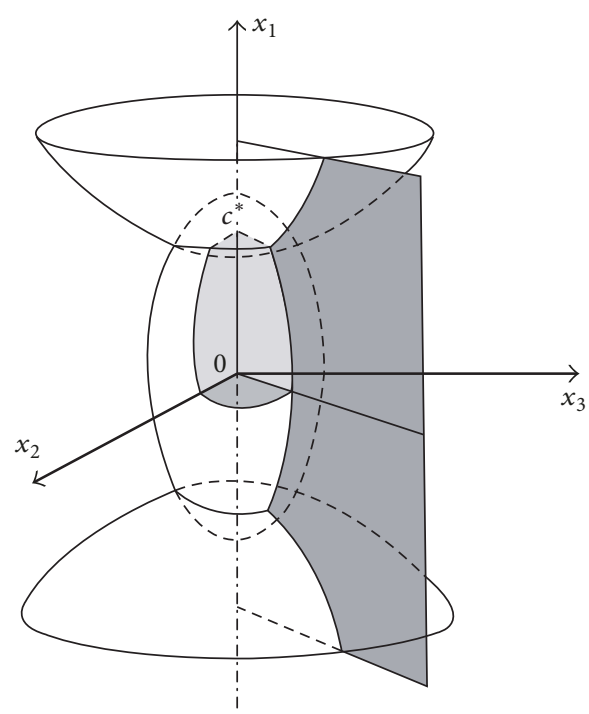

FIgURE 1: The prolate spheroidal geometry and the three coordinate surfaces, that is, prolate spheroids ( $\tau=$ const.), hyperboloids of two sheets $(\zeta=$ const.), and meridian planes ( $\varphi=$ const.) with $c$ being the semifocal distance of the coordinate system.

$\tau \rightarrow+\infty$ it approaches a sphere located at infinity. A key point in this analysis is the exploitation of the common $x_{1}$ axis of symmetry between the applicable coordinate system and the spheroidal body under investigation. In fact, this is a quite practical technique, which simplifies the mathematical treatment, though it retains the generality unaltered and we still cope with a genuine three-dimensional problem, since the arbitrary orientation of the magnetic dipole, which is implied by (2), defends the $3 \mathrm{D}$ configuration until the final solution. Nevertheless, in the very special case where the magnetic dipole is set on the axis of symmetry of the proposed prolate geometry, that is, the $x_{1}$-axis, which means $\mathbf{r}_{0}=\left(x_{10}, 0,0\right)$ and is orientated to the $x_{1}$-direction, yielding $\mathbf{m}=m_{1} \widehat{\mathbf{x}}_{1}$, then the problem under consideration turns into being axisymmetric, since any kind of dependence from the azimuthal angular $\varphi \in[0,2 \pi)$ is omitted. This restriction is imposed in every field in addition to the vanishing of the other two components of the magnetic dipole $\left(m_{2}=m_{3}=0\right)$ and of its position $\left(x_{20}=x_{30}=0\right)$ to the analytical results. However, the general nonaxisymmetric case that is examined here is concerned with a source of arbitrary location and orientation.

The main differential operators, in the prolate geometry, excluding the tip for $\tau \rightarrow 1^{+}$and for every $\tau \in(1,+\infty), \zeta \in$ $[-1,1]$, and $\varphi \in[0,2 \pi)$, are furnished by

$$
\begin{aligned}
\nabla_{\mathbf{r}} \equiv & \nabla \\
= & \frac{1}{c \sqrt{\tau^{2}-\zeta^{2}}}\left[\sqrt{\tau^{2}-1} \widehat{\boldsymbol{\tau}} \frac{\partial}{\partial \tau}-\sqrt{1-\zeta^{2}} \widehat{\zeta} \frac{\partial}{\partial \zeta}\right] \\
& +\frac{1}{c \sqrt{\tau^{2}-1} \sqrt{1-\zeta^{2}}} \widehat{\boldsymbol{\varphi}} \frac{\partial}{\partial \varphi}
\end{aligned}
$$


as for the gradient and

$$
\begin{aligned}
\Delta_{\mathbf{r}} & \equiv \Delta=\frac{1}{c^{2}\left(\tau^{2}-\zeta^{2}\right)}\left\{\frac{\partial}{\partial \tau}\left[\left(\tau^{2}-1\right) \frac{\partial}{\partial \tau}\right]\right. \\
& \left.+\frac{\partial}{\partial \zeta}\left[\left(1-\zeta^{2}\right) \frac{\partial}{\partial \zeta}\right]\right\}+\frac{1}{c^{2}\left(\tau^{2}-1\right)\left(1-\zeta^{2}\right)} \frac{\partial^{2}}{\partial \varphi^{2}},
\end{aligned}
$$

concerning the Laplacian. In (34), the outward unit normal vector coincides with $\widehat{\boldsymbol{\tau}}$ through

$$
\begin{aligned}
\widehat{\mathbf{n}} \equiv & \widehat{\boldsymbol{\tau}}(\tau, \zeta, \varphi)=\frac{1}{\sqrt{\tau^{2}-\zeta^{2}}}\left(\zeta \sqrt{\tau^{2}-1} \widehat{\mathbf{x}}_{1}\right. \\
& \left.+\tau \sqrt{1-\zeta^{2}} \cos \varphi \widehat{\mathbf{x}}_{2}+\tau \sqrt{1-\zeta^{2}} \sin \varphi \widehat{\mathbf{x}}_{3}\right)
\end{aligned}
$$

whereas the other two orthonormal vectors of the spheroidal system assume the Cartesian basis formulae

$$
\begin{aligned}
& \widehat{\zeta}(\tau, \zeta, \varphi)=\frac{1}{\sqrt{\tau^{2}-\zeta^{2}}}\left(-\tau \sqrt{1-\zeta^{2}} \widehat{\mathbf{x}}_{1}\right. \\
& \left.\quad+\zeta \sqrt{\tau^{2}-1} \cos \varphi \widehat{\mathbf{x}}_{2}+\zeta \sqrt{\tau^{2}-1} \sin \varphi \widehat{\mathbf{x}}_{3}\right), \\
& \widehat{\boldsymbol{\varphi}}(\varphi)=-\sin \varphi \widehat{\mathbf{x}}_{2}+\cos \varphi \widehat{\mathbf{x}}_{3},
\end{aligned}
$$

while the unit dyadic in prolate spheroidal coordinates is $\widetilde{\mathbf{I}}=$ $\widehat{\boldsymbol{\tau}} \otimes \widehat{\boldsymbol{\tau}}+\widehat{\zeta} \otimes \widehat{\zeta}+\widehat{\boldsymbol{\varphi}} \otimes \widehat{\boldsymbol{\varphi}}$

Specifying the solid surface $S$ by $\tau=\tau_{s} \equiv a_{1} / c$, then the prolate spheroidal body has major (in $x_{1}$-direction) and minor (situated on $x_{2} x_{3}$-plane) axes $a_{1}=c \tau_{s}$ and $a_{2}=a_{3}=$ $c \sqrt{\tau_{s}^{2}-1}$, respectively, while its eccentricity is $e=1 / \tau_{s}$. We remark that the low-frequency approximations are secured only when the main axes, as well as the semifocal distance, are less than the wavelength $\lambda=2 \pi / k$ of the incident field, such a demand being ensured via the inequality $2 c+a_{1}+a_{2}+a_{3} \ll$ $\lambda$. In terms of spheroidal modes, the actual region of wave propagation is

$$
\begin{aligned}
\Omega & \equiv V\left(\mathbb{R}^{3}\right)-\left\{\mathbf{r}_{0}\right\} \\
= & \left\{(\tau, \zeta, \varphi): \tau \in\left[\tau_{s},+\infty\right), \quad \zeta \in[-1,1], \varphi\right. \\
& \in[0,2 \pi)\}-\left\{\left(\tau_{0}, \zeta_{0}, \varphi_{0}\right)\right\},
\end{aligned}
$$

where $\left(\tau_{0}, \zeta_{0}, \varphi_{0}\right)$ corresponds to $\left(x_{10}, x_{20}, x_{30}\right)$ by means of (33), forming an exterior domain. Apparently, since the spheroidal object is impenetrable, it lacks any field inside; that is,

$$
\begin{aligned}
& \mathbf{H}^{x}(\mathbf{r})=\mathbf{E}^{x}(\mathbf{r})=\mathbf{0} \\
& \text { for } \mathbf{r} \in\left\{\tau \in\left(1, \tau_{s}\right), \zeta \in[-1,1], \varphi \in[0,2 \pi)\right\}, \text { with } x=p, s, t,
\end{aligned}
$$

while on $\tau=\tau_{s}$ and as $\tau \rightarrow+\infty$, the boundary conditions (23) or (24) and the limiting behavior (25) or (26), respectively, must be counted.

Proceeding to the analysis, we introduce the interior $u_{\ell, \text { in }}^{m / q}$ (regular as $\tau \rightarrow 1^{+}$) and the exterior $u_{\ell \text {,ex }}^{m / q}$ (regular as $\tau \rightarrow$ $+\infty)$ prolate spheroidal harmonic eigenfunctions of degree $\ell \geq 0$ and of order $m=0,1,2, \ldots, \ell$, in terms of the associated Legendre functions of the first $P_{\ell}^{m}$ and of the second $Q_{\ell}^{m}$ kind (for more information about these functions and their properties, refer to $[7,14,15]$, where a detailed appendix contains such details) according to

$$
\begin{aligned}
& u_{\ell, \mathrm{in}}^{m / q}(\mathbf{r})=P_{\ell}^{m}(\tau) P_{\ell}^{m}(\zeta) f_{m}^{q}(\varphi), \\
& u_{\ell, \mathrm{ex}}^{m / q}(\mathbf{r})=Q_{\ell}^{m}(\tau) P_{\ell}^{m}(\zeta) f_{m}^{q}(\varphi),
\end{aligned}
$$

respectively, and for any $\tau \in[1,+\infty), \zeta \in[-1,1]$, and $\varphi \in$ $[0,2 \pi)$, which both are regular on the axis of symmetry. The azimuthal angular dependence reads

$$
\begin{array}{r}
f_{m}^{q}(\varphi)=\left\{\begin{array}{ll}
\cos m \varphi, & q=e \\
\sin m \varphi, & q=o
\end{array} \Longrightarrow\right. \\
f_{m}^{q^{\prime}}(\varphi)= \begin{cases}-m \sin m \varphi, & q=e \\
m \cos m \varphi, & q=o\end{cases}
\end{array}
$$

for every $\varphi \in[0,2 \pi)$,

where $q$ stands for the even $(q=e)$ or the odd $(q=o)$ part of the eigenfunctions, while the prime denotes derivation with respect to the argument here and thereafter. Consequently, every harmonic function $u$ in prolate spheroidal geometry belongs to the kernel space of Laplace's operator (35), that is, $\Delta u=0$, and assumes

$$
\begin{aligned}
& u(\mathbf{r})=\sum_{\ell=0}^{+\infty} \sum_{m=0}^{\ell} \sum_{q=e, o}\left[A_{\ell, \mathrm{in}}^{m / q} u_{\ell, \mathrm{in}}^{m / q}(\mathbf{r})+A_{\ell, \mathrm{ex}}^{m / q} u_{\ell, \mathrm{ex}}^{m / q}(\mathbf{r})\right] \\
& \text { for } \mathbf{r} \in\{\tau \in[1,+\infty), \zeta \in[-1,1], \varphi \in[0,2 \pi)\},
\end{aligned}
$$

where $A_{\ell \text {,in }}^{m / q}$ and $A_{\ell, \text { ex }}^{m / q}$ are arbitrary constants, which selectively vanish once we deal with either an interior problem $\left(A_{\ell, \mathrm{ex}}^{m / q}=0\right)$ or an exterior one $\left(A_{\ell, \text { in }}^{m / q}=0\right)$, like in our case, in order to obtain the mathematically accepted solution. On the other hand, we join the internal product

$$
\begin{aligned}
\left(P_{\ell}^{m}(\zeta), P_{\ell^{\prime}}^{m}(\zeta)\right) & =\int_{-1}^{+1} P_{\ell}^{m}(\zeta) P_{\ell^{\prime}}^{m}(\zeta) d \zeta \\
& =\frac{2}{2 \ell+1} \frac{(\ell+m) !}{(\ell-m) !} \delta_{\ell \ell^{\prime}}
\end{aligned}
$$

with $\ell, \ell^{\prime} \geq 0$,

needed in our project, $\delta_{\ell \ell^{\prime}}$ being the Kronecker delta and $m=$ $0,1,2, \ldots, \ell$, while the trigonometric functions for $\ell, \ell^{\prime} \geq 0$ and $q, q^{\prime}=e, o$ are orthogonal with respect to

$$
\begin{aligned}
\left(f_{m}^{q}(\varphi), f_{m^{\prime}}^{q^{\prime}}(\varphi)\right) & =\int_{0}^{2 \pi} f_{m}^{q}(\varphi) f_{m^{\prime}}^{q^{\prime}}(\varphi) d \varphi \\
& =\frac{2 \pi}{\varepsilon_{m}} \delta_{m m^{\prime}} \delta_{q q^{\prime}} \\
& \text { with }\left(m, m^{\prime}\right)=0,1,2, \ldots,\left(\ell, \ell^{\prime}\right),
\end{aligned}
$$


where $\delta_{m m^{\prime}}$ and $\delta_{q q^{\prime}}$ are again the Kronecker deltas with $\varepsilon_{0}=$ 1 and $\varepsilon_{m}=2$ otherwise.

Furthermore, a very crucial piece of information for the forthcoming analytical subsections incorporates the position of the dipole at $\mathbf{r}_{0}$ with the very useful Green's function expansion for prolate geometry [14], so that in terms of (41) and for any observation region (39), we obtain

$$
\begin{aligned}
\frac{1}{R} \equiv & \frac{1}{\left|\mathbf{r}-\mathbf{r}_{0}\right|} \\
& = \begin{cases}\sum_{\ell=0}^{+\infty} \sum_{m=0}^{\ell} \sum_{q=e, o} \rho_{\ell, \text { in }}^{m / q}\left(\mathbf{r}_{0}\right) u_{\ell, \mathrm{ex}}^{m / q}(\mathbf{r}) & \text { for } \mathbf{r}>\mathbf{r}_{0} \\
\sum_{\ell=0}^{+\infty} \sum_{m=0}^{\ell} \sum_{q=e, o} \rho_{\ell, \mathrm{ex}}^{m / q}\left(\mathbf{r}_{0}\right) u_{\ell, \mathrm{in}}^{m / q}(\mathbf{r}) & \text { for } \mathbf{r}<\mathbf{r}_{0},\end{cases}
\end{aligned}
$$

where at $\mathbf{r}=\mathbf{r}_{0}=\left(\tau_{0}, \zeta_{0}, \varphi_{0}\right)$ we attain singularity and it holds

$$
\rho_{\ell, y}^{m / q}\left(\mathbf{r}_{0}\right)=\frac{(2 \ell+1)}{c}\left[\frac{(\ell-m) !}{(\ell+m) !}\right]^{2}(-1)^{m} \varepsilon_{m} u_{\ell, y}^{m / q}\left(\mathbf{r}_{0}\right)
$$

with $y=$ in, ex,

which ends the well-known, but necessary, gathering of preliminaries for the sequel.

Finally, for completeness, we invoke the exact expressions of gradient action upon interior and exterior solid spheroidal harmonics (41) via the surface ones for the shake of clarity. This is feasible, since $\nabla u_{\ell \text {,in }}^{m / q}$ and $\nabla u_{\ell, \text { ex }}^{m / q}$ for $\ell \geq 0, m=$ $0,1,2, \ldots, \ell$, and $q=e, o$ belong to the subspace produced by the surface spheroidal harmonics. After cumbersome analysis, profiting by recurrence relations for $P_{\ell}^{m}$ and $Q_{\ell}^{m}$, defined for different arguments, that is, for $\tau \geq 1$ and $|\zeta| \leq 1$ $[14,15]$, we arrive at

$$
\begin{aligned}
& \nabla u_{\ell, \text { in }}^{m / q}(\mathbf{r}) \\
& =\frac{f_{m}^{q}(\varphi)}{(2 \ell+1)}\left\{\frac{P_{\ell}^{m}(\zeta) \widehat{\boldsymbol{\tau}}}{c \sqrt{\tau^{2}-\zeta^{2}} \sqrt{\tau^{2}-1}}[\ell(\ell-m+1)\right. \\
& \left.\cdot P_{\ell+1}^{m}(\tau)-(\ell+1)(\ell+m) P_{\ell-1}^{m}(\tau)\right] \\
& +\frac{P_{\ell}^{m}(\tau) \widehat{\zeta}}{c \sqrt{\tau^{2}-\zeta^{2}} \sqrt{1-\zeta^{2}}}\left[\ell(\ell-m+1) P_{\ell+1}^{m}(\zeta)\right.
\end{aligned}
$$

$$
\begin{aligned}
& \left.\left.-(\ell+1)(\ell+m) P_{\ell-1}^{m}(\zeta)\right]\right\}+\frac{f_{m}^{q^{\prime}}(\varphi) \hat{\boldsymbol{\varphi}}}{c \sqrt{\tau^{2}-1} \sqrt{1-\zeta^{2}}} \\
& \cdot P_{\ell}^{m}(\tau) P_{\ell}^{m}(\zeta) \\
& \quad \text { for } \ell \geq 0, m=0,1,2, \ldots, \ell, q=e, o,
\end{aligned}
$$

as far as the gradient on the interior solid harmonics is concerned, while

$$
\begin{aligned}
& \nabla u_{\ell, \mathrm{ex}}^{m / q}(\mathbf{r}) \\
& =\frac{f_{m}^{q}(\varphi)}{(2 \ell+1)}\left\{\frac{P_{\ell}^{m}(\zeta) \widehat{\boldsymbol{\tau}}}{c \sqrt{\tau^{2}-\zeta^{2}} \sqrt{\tau^{2}-1}}[\ell(\ell-m+1)\right. \\
& \left.\cdot Q_{\ell+1}^{m}(\tau)-(\ell+1)(\ell+m) Q_{\ell-1}^{m}(\tau)-\delta_{\ell 0}\right] \\
& +\frac{Q_{\ell}^{m}(\tau) \widehat{\zeta}}{c \sqrt{\tau^{2}-\zeta^{2}} \sqrt{1-\zeta^{2}}}\left[\ell(\ell-m+1) P_{\ell+1}^{m}(\zeta)\right. \\
& \left.\left.-(\ell+1)(\ell+m) P_{\ell-1}^{m}(\zeta)\right]\right\}+\frac{f_{m}^{q^{\prime}}(\varphi) \widehat{\varphi}}{c \sqrt{\tau^{2}-1} \sqrt{1-\zeta^{2}}} \\
& \cdot Q_{\ell}^{m}(\tau) P_{\ell}^{m}(\zeta) \\
& \quad \text { for } \ell \geq 0, m=0,1,2, \ldots, \ell, q=e, o,
\end{aligned}
$$

regarding the gradient on the exterior solid harmonics. Both (48) and (49) are defined for any $\tau \in(1,+\infty), \zeta \in[-1,1]$, and $\varphi \in[0,2 \pi)$, while $\delta_{\ell 0}=1$ only when $\ell=m=0$; else it is zero. It is obvious that expressions (48) and (49) are also applicable at $\mathbf{r}=\mathbf{r}_{0}$, if we make the replacement $\nabla \rightarrow \nabla_{\mathbf{r}_{0}}$, whereas then the prime declares derivation with respect to $\varphi_{0}$.

Next, we perform long and tedious calculations in order to pursuit an analytical form for the scattered fields. In what follows, we present the basic and interesting steps of them by starting from the magnetic modes to obtain $\mathbf{H}_{0}^{s}, \mathbf{H}_{2}^{s}$, and $\mathbf{H}_{3}^{s}$ and proceed to the electric ones $\mathbf{E}_{1}^{s}, \mathbf{E}_{3}^{s}$, bearing in mind the vanishing electromagnetic terms $\mathbf{H}_{1}^{\mathcal{S}}=\mathbf{E}_{0}^{\mathcal{S}}=\mathbf{E}_{2}^{\mathcal{S}}=\mathbf{0}$, where $\mathbf{H}_{2}^{s}$ and $\mathbf{E}_{3}^{s}$ are the most cumbersome cases, which we endeavor to retrieve in a handy form.

3.2. The $\mathbf{H}_{0}^{s}, \mathbf{H}_{2}^{s}$, and $\mathbf{H}_{3}^{s}$ Magnetic Fields. Beginning with the situation for the Rayleigh static term, the relative potential boundary value problem that has to be solved embodies (27) and (28) with the Neumann boundary condition (24) for $n=$ 0 , yielding

$$
\mathbf{H}_{0}^{s}(\mathbf{r})=\nabla \Phi_{0}^{s}(\mathbf{r}) \quad \text { with } \Delta \Phi_{0}^{s}(\mathbf{r})=0 \text { for } \mathbf{r} \in \Omega \text {, where } \widehat{\boldsymbol{\tau}}\left(\mathbf{r}_{s}\right) \cdot\left[\mathbf{H}_{0}^{p}\left(\mathbf{r}_{s}\right)+\mathbf{H}_{0}^{s}\left(\mathbf{r}_{s}\right)\right]=0
$$

with $\mathbf{r}_{s}=\left(\tau_{s}, \zeta, \varphi\right)$, where $\widehat{\boldsymbol{\tau}}$ is provided by (36) for $\tau=$ $\tau_{s}$. The harmonic potential $\Phi_{0}^{s}$ is rendered via the exteriortype part of (43); hence, by virtue of (50), we conclude to

$$
\mathbf{H}_{0}^{s}(\mathbf{r})=\sum_{\ell=0}^{+\infty} \sum_{m=0}^{\ell} \sum_{q=e, o} a_{\ell, \mathrm{ex}}^{m / q} \nabla\left[Q_{\ell}^{m}(\tau) P_{\ell}^{m}(\zeta) f_{m}^{q}(\varphi)\right]
$$

for every $\mathbf{r} \in \Omega$, 
where $a_{\ell, \mathrm{ex}}^{m / q}$ for $\ell \geq 0, m=0,1,2, \ldots, \ell$, and $q=$ $e, o$ denote the constant coefficients to be recovered from boundary condition (50). Expansion (51) is compatible with the acquired limiting behavior (26). In the first place, we imply definition (34) upon (51) and we calculate the inner product

$$
\begin{aligned}
& \widehat{\boldsymbol{\tau}}\left(\tau_{s}, \zeta, \varphi\right) \cdot \mathbf{H}_{0}^{s}\left(\tau_{s}, \zeta, \varphi\right)=\frac{\sqrt{\tau_{s}^{2}-1}}{c \sqrt{\tau_{s}^{2}-\zeta^{2}}} \\
& \quad \cdot \sum_{\ell=0}^{+\infty} \sum_{m=0}^{\ell} \sum_{q=e, o} a_{\ell, \mathrm{ex}}^{m / q}\left[Q_{\ell}^{m^{\prime}}\left(\tau_{s}\right) P_{\ell}^{m}(\zeta) f_{m}^{q}(\varphi)\right]
\end{aligned}
$$

for every $\zeta \in[-1,1]$ and $\varphi \in[0,2 \pi)$. On the other hand, the corresponding inner product with the incident field (7) encounters a complexity, caused by the double action of the gradient operator over Green's function quantity. Aiming to exceed this difficultness, we work as follows. Firstly, we take the unit normal projection of (34), where the outcome is

$$
\begin{aligned}
& \widehat{\boldsymbol{\tau}}\left(\tau_{s}, \zeta, \varphi\right) \cdot \mathbf{H}_{0}^{p}\left(\tau_{s}, \zeta, \varphi ; \mathbf{r}_{0}\right) \\
& =\frac{\sqrt{\tau_{s}^{2}-1}}{c \sqrt{\tau_{s}^{2}-\zeta^{2}}}\left\{\frac{\partial}{\partial \tau}\left[\nabla \frac{1}{\left|\mathbf{r}-\mathbf{r}_{0}\right|}\right] \cdot \frac{\mathbf{m}}{4 \pi}\right\}_{\tau=\tau_{s}},
\end{aligned}
$$

since the dyadic $\nabla \otimes \nabla(1 / R)$ is symmetric, and we employ (12) to rewrite (53) as

$$
\begin{aligned}
& \widehat{\boldsymbol{\tau}}\left(\tau_{s}, \zeta, \varphi\right) \cdot \mathbf{H}_{0}^{p}\left(\tau_{s}, \zeta, \varphi ; \mathbf{r}_{0}\right) \\
& \quad=-\frac{\sqrt{\tau_{s}^{2}-1}}{c \sqrt{\tau_{s}^{2}-\zeta^{2}}}\left\{\frac{\partial}{\partial \tau}\left[\nabla_{\mathbf{r}_{0}} \frac{1}{\left|\mathbf{r}-\mathbf{r}_{0}\right|}\right] \cdot \frac{\mathbf{m}}{4 \pi}\right\}_{\tau=\tau_{s}},
\end{aligned}
$$

which, upon introduction of eigenexpansion (46) with (47) for $\mathbf{r}=\mathbf{r}_{s}<\mathbf{r}_{0}$, becomes

$$
\begin{aligned}
& \widehat{\boldsymbol{\tau}}\left(\mathbf{r}_{s}\right) \cdot \mathbf{H}_{0}^{p}\left(\mathbf{r}_{s} ; \mathbf{r}_{0}\right)=-\frac{\sqrt{\tau_{s}^{2}-1}}{c \sqrt{\tau_{s}^{2}-\zeta^{2}}} \\
& \quad \cdot \sum_{\ell=0}^{+\infty} \sum_{m=0}^{\ell} \sum_{q=e, 0}\left[\frac{\mathbf{m}}{4 \pi} \cdot \nabla_{\mathbf{r}_{0}} \rho_{\ell, \mathrm{ex}}^{m / q}\left(\mathbf{r}_{0}\right)\right] P_{\ell}^{m^{\prime}}\left(\tau_{s}\right) P_{\ell}^{m}(\zeta) \\
& \cdot f_{m}^{q}(\varphi),
\end{aligned}
$$

where $\rho_{\ell, \mathrm{ex}}^{m / q}\left(\mathbf{r}_{0}\right)$ that has to do with the source is defined in (47) and $\mathbf{r}_{s}=\left(\tau_{s}, \zeta, \varphi\right)$, while $\zeta \in[-1,1]$ and $\varphi \in[0,2 \pi)$. Apparently, the gradient $\nabla_{\mathbf{r}_{0}} \rho_{\ell, \mathrm{ex}}^{m / q}\left(\mathbf{r}_{0}\right)$ is a known quantity and $\mathbf{m}$ satisfies (2). Combining (52) and (55), condition (50) reveals that

$$
\begin{aligned}
& \frac{\sqrt{\tau_{s}^{2}-1}}{c \sqrt{\tau_{s}^{2}-\zeta^{2}}} \\
& \cdot \sum_{\ell=0}^{\infty} \sum_{m=0}^{\ell} \sum_{q=e, o}\left[a_{\ell, \mathrm{ex}}^{m / q} Q_{\ell}^{m^{\prime}}\left(\tau_{s}\right)-\left(\frac{\mathbf{m}}{4 \pi} \cdot \nabla_{\mathbf{r}_{0}} \rho_{\ell, \mathrm{ex}}^{m / q}\left(\mathbf{r}_{0}\right)\right) P_{\ell}^{m^{\prime}}\left(\tau_{s}\right)\right] \\
& \cdot P_{\ell}^{m}(\zeta) f_{m}^{q}(\varphi)=0
\end{aligned}
$$

at any $\zeta \in[-1,1]$ and $\varphi \in[0,2 \pi)$. Orthogonality arguments based on (44) and (45) lead to the obvious and compact result

$$
a_{\ell, \mathrm{ex}}^{m / q}=\left(\frac{\mathbf{m}}{4 \pi} \cdot \nabla_{\mathbf{r}_{0}} \rho_{\ell, \mathrm{ex}}^{m / q}\left(\mathbf{r}_{0}\right)\right) \frac{P_{\ell}^{m^{\prime}}\left(\tau_{s}\right)}{Q_{\ell}^{m^{\prime}}\left(\tau_{s}\right)}
$$

$$
\text { for } \ell \geq 0, m=0,1,2, \ldots, \ell, q=e, o
$$

at the singularity point $\mathbf{r}_{0}=\left(\tau_{0}, \zeta_{0}, \varphi_{0}\right)$. Substituting relationship (57) into (51), we reach the sought solution, that is,

$$
\begin{gathered}
\mathbf{H}_{0}^{s}(\mathbf{r})=\frac{\mathbf{m}}{4 \pi c} \cdot \sum_{\ell=0}^{+\infty} \sum_{m=0}^{\ell} \sum_{q=e, o}(2 \ell+1)\left[\frac{(\ell-m) !}{(\ell+m) !}\right]^{2} \\
\cdot(-1)^{m} \varepsilon_{m} \frac{P_{\ell}^{m^{\prime}}\left(\tau_{s}\right)}{Q_{\ell}^{m^{\prime}}\left(\tau_{s}\right)}\left[\nabla_{\mathbf{r}_{0}} u_{\ell, \mathrm{ex}}^{m / q}\left(\mathbf{r}_{0}\right) \otimes \nabla u_{\ell, \mathrm{ex}}^{m / q}(\mathbf{r})\right]
\end{gathered}
$$

for every $\mathbf{r} \in \Omega$, whereas $u_{\ell \text {, ex }}^{m / q}$ and $u_{\ell, \text { ex }}^{m / q}\left(\mathbf{r}_{0}\right)$ are provided via the right-hand side formulae of (41), while $\nabla u_{\ell, \text { ex }}^{m / q}$ arises from (49), the same thing for $\nabla_{\mathbf{r}_{0}} u_{\ell, \mathrm{ex}}^{m / q}\left(\mathbf{r}_{0}\right)$, by a simple switch of arguments, all written for $\ell \geq 0, m=0,1,2, \ldots, \ell$, and $q=e, o$.

In the sequel, we treat the magnetic field-case for $n=$ 2 , which exhibits a particular difficulty, mainly due to the coupling with the static term, indicated by (29). However, secondarily speaking, since $\mathbf{H}_{0}^{s}$ is a pure vector field, needing a set of three separate boundary conditions to be determined, then, beyond the application of the scalar condition in the first part of (24) at $n=2$, it is indirectly associated with the total electric field $\mathbf{E}_{1}^{t}$ on the boundary through the second part of (24) by implying (10) and (30). All the above are mathematically summarized as follows:

$$
\begin{aligned}
& \mathbf{H}_{2}^{s}(\mathbf{r})=\mathbf{X}_{2}^{s}(\mathbf{r})+\mathbf{r} \Phi_{0}^{s}(\mathbf{r}) \\
& \quad \text { with } \Delta \mathbf{X}_{2}^{s}(\mathbf{r})=\mathbf{0}, \Delta \Phi_{0}^{s}(\mathbf{r})=0 \text { for } \mathbf{r} \in \Omega,
\end{aligned}
$$

where, provided $\mathbf{H}_{2}^{p}$ directly from (8) and given (46) with (47),

$$
\begin{aligned}
& \widehat{\boldsymbol{\tau}}(\mathbf{r}) \cdot\left[\mathbf{H}_{2}^{p}(\mathbf{r})+\mathbf{H}_{2}^{s}(\mathbf{r})\right]=0, \\
& \widehat{\boldsymbol{\tau}}(\mathbf{r}) \times\left[\frac{\mathbf{m}}{2 \pi} \times \nabla \frac{1}{R}+\nabla \times \mathbf{H}_{2}^{s}(\mathbf{r})\right]=\mathbf{0}, \\
& \text { when } \mathbf{r}=\mathbf{r}_{s}=\left(\tau_{s}, \zeta, \varphi\right),
\end{aligned}
$$

which completes the boundary value problem that has to be solved for this situation, by the imposition of the divergencefree property of $\mathbf{H}_{2}^{s}$ that must be always valid via

$$
\begin{aligned}
\nabla \cdot \mathbf{H}_{2}^{s}(\mathbf{r}) & =0 \Longrightarrow \\
\nabla \cdot \mathbf{X}_{2}^{s}(\mathbf{r})+(3+\mathbf{r} \cdot \nabla) \Phi_{0}^{s}(\mathbf{r}) & =0,
\end{aligned}
$$


Inserting, now, (57) into (51) and combining it with the fact that $\mathbf{H}_{0}^{s}=\nabla \Phi_{0}^{s}$, then the zeroth-order potential is known and admits

$$
\begin{gathered}
\Phi_{0}^{s}(\mathbf{r})=\sum_{\ell=0}^{+\infty} \sum_{m=0}^{\ell} \sum_{q=e, o}\left(\frac{\mathbf{m}}{4 \pi} \cdot \nabla_{\mathbf{r}_{0}} \rho_{\ell, \mathrm{ex}}^{m / q}\left(\mathbf{r}_{0}\right)\right) \\
\cdot \frac{P_{\ell}^{m^{\prime}}\left(\tau_{s}\right)}{Q_{\ell}^{m^{\prime}}\left(\tau_{s}\right)}\left[Q_{\ell}^{m}(\tau) P_{\ell}^{m}(\zeta) f_{m}^{q}(\varphi)\right] \quad \text { for } \mathbf{r} \in \Omega,
\end{gathered}
$$

whereas $\rho_{\ell, \text { ex }}^{m / q}\left(\mathbf{r}_{0}\right)$ for $\ell \geq 0, m=0,1,2, \ldots, \ell$, and $q=e, o$ follow from (47). On the other hand, the vector harmonic function $\mathbf{X}_{2}^{s}$ keeps the exterior-type solution fashion, leading to

$$
\mathbf{X}_{2}^{s}(\mathbf{r})=\sum_{\ell=0}^{+\infty} \sum_{m=0}^{\ell} \sum_{q=e, o} \mathbf{b}_{\ell, \mathrm{ex}}^{m / q}\left[Q_{\ell}^{m}(\tau) P_{\ell}^{m}(\zeta) f_{m}^{q}(\varphi)\right]
$$

for every $\mathbf{r} \in \Omega$,

where (63) meets the radiation criteria imposed by (26), while

$$
\begin{gathered}
\mathbf{b}_{\ell, \mathrm{ex}}^{m / q}=\sum_{j=1}^{3} b_{\ell, j}^{m / q} \widehat{\mathbf{x}}_{j}=b_{\ell, 1}^{m / q} \widehat{\mathbf{x}}_{1}+b_{\ell, 2}^{m / q} \widehat{\mathbf{x}}_{2}+b_{\ell, 3}^{m / q} \widehat{\mathbf{x}}_{3}=\left(\tau^{2}\right. \\
\left.-\zeta^{2}\right)^{-1 / 2}\left\{\left(b_{\ell, 1}^{m / q} \zeta \sqrt{\tau^{2}-1}+b_{\ell, 2}^{m / q} \tau \sqrt{1-\zeta^{2}} \cos \varphi\right.\right. \\
\left.+b_{\ell, 3}^{m / q} \tau \sqrt{1-\zeta^{2}} \sin \varphi\right) \widehat{\boldsymbol{\tau}}+\left(-b_{\ell, 1}^{m / q} \tau \sqrt{1-\zeta^{2}}\right. \\
\left.\left.+b_{\ell, 2}^{m / q} \zeta \sqrt{\tau^{2}-1} \cos \varphi+b_{\ell, 3}^{m / q} \zeta \sqrt{\tau^{2}-1} \sin \varphi\right) \widehat{\zeta}\right\} \\
+\left(-b_{\ell, 2}^{m / q} \sin \varphi+b_{\ell, 3}^{m / q} \cos \varphi\right) \widehat{\varphi} \\
\quad \text { for } \ell \geq 0, m=0,1,2, \ldots, \ell, q=e, o
\end{gathered}
$$

denote the vector character of the unknown constant coefficients $\mathbf{b}_{\ell \text {,ex }}^{m / q}$, while, willing to be consistent with (64) and work within the frame of purely prolate spheroidal geometry, we are obliged to write the spheroidal representation of the position vector, entering (59) as

$$
\mathbf{r}=\sum_{j=1}^{3} x_{j} \widehat{\mathbf{x}}_{j}=\frac{c}{\sqrt{\tau^{2}-\zeta^{2}}}\left(\tau \sqrt{\tau^{2}-1} \widehat{\boldsymbol{\tau}}-\zeta \sqrt{1-\zeta^{2}} \widehat{\zeta}\right)
$$

$$
\text { for every } \tau \in(1,+\infty), \zeta \in[-1,1] \text {. }
$$

The field problem under consideration has an additional physical difficulty, which is inherited from the corresponding mathematical complexity. Thus, in what follows, we give emphasis on the main steps due to the large number of calculations to perform. To this end, we handle (60) in such a manner so as to obtain three separate sets of boundary conditions, one from the $\tau$-component and two from rela- tions, which correspond to the $\zeta, \varphi$-components. This is accomplished by operating $\widehat{\boldsymbol{\tau}}$ from (36) into (60) and tackling with (46) for $\tau=\tau_{s}<\tau_{0}$. Once done, we reach cumbersomely the following three relations for the coefficients, satisfying

$$
\begin{aligned}
& \sum_{\ell=0}^{+\infty} \sum_{m=0}^{\ell} \sum_{q=e, o}\left[\sum_{j=1}^{3} f_{\ell, j}^{m / q, \kappa}\left(\tau_{s}, \zeta, \varphi\right) b_{\ell, j}^{m / q}-g_{\ell}^{m / q, \kappa}\left(\tau_{s}, \zeta, \varphi ; \mathbf{r}_{0}\right)\right] \\
& =0 \quad \text { for any } \kappa=1,2,3,
\end{aligned}
$$

counting for the aforementioned three sets of boundary relations, where $\zeta \in[-1,1], \varphi \in[0,2 \pi)$, and $\mathbf{r}_{0}=\left(\tau_{0}, \zeta_{0}, \varphi_{0}\right)$. Omitting dependence on $\left(\tau_{s}, \zeta, \varphi\right)$ and $\left(\tau_{s}, \zeta, \varphi ; \mathbf{r}_{0}\right)$ for reasons of writing clarity, the leading functions of the unknown constant coefficients and those comprising the constant terms of (66) are furnished initially for $\kappa=1$ by the formulae

$$
\begin{aligned}
& f_{\ell, 1}^{m / q, 1}=c \sqrt{\tau_{s}^{2}-1} Q_{\ell}^{m}\left(\tau_{s}\right) \zeta P_{\ell}^{m}(\zeta) f_{m}^{q}(\varphi), \\
& f_{\ell, 2}^{m / q, 1}=c \tau_{s} Q_{\ell}^{m}\left(\tau_{s}\right) \sqrt{1-\zeta^{2}} P_{\ell}^{m}(\zeta) \cos \varphi f_{m}^{q}(\varphi), \\
& f_{\ell, 3}^{m / q, 1}=c \tau_{s} Q_{\ell}^{m}\left(\tau_{s}\right) \sqrt{1-\zeta^{2}} P_{\ell}^{m}(\zeta) \sin \varphi f_{m}^{q}(\varphi), \\
& g_{\ell}^{m / q, 1}=\left\{\rho_{\ell, \mathrm{ex}}^{m / q}\left(\mathbf{r}_{0}\right) \frac{\mathbf{m}}{4 \pi}\right. \\
& \cdot\left[-\sqrt{\tau_{s}^{2}-1} P_{\ell}^{m^{\prime}}\left(\tau_{s}\right)\left(\mathbf{r}\left(\tau_{s}, \zeta, \varphi\right)-\mathbf{r}_{0}\right)\right. \\
& \left.\quad+c \sqrt{\tau_{s}^{2}-\zeta^{2}} P_{\ell}^{m}\left(\tau_{s}\right) \hat{\boldsymbol{\tau}}\right]-c^{2} M_{\ell, \mathrm{ex}}^{m / q}\left(\mathbf{r}_{0}\right) \\
& \left.\quad \cdot \tau_{s} \sqrt{\tau_{s}^{2}-1}\left[\frac{Q_{\ell}^{m}\left(\tau_{s}\right)}{Q_{\ell}^{m^{\prime}}\left(\tau_{s}\right)}\right] P_{\ell}^{m^{\prime}}\left(\tau_{s}\right)\right\} P_{\ell}^{m}(\zeta) f_{m}^{q}(\varphi),
\end{aligned}
$$

while for $\kappa=2$ it is readily obtained

$$
\begin{aligned}
& f_{\ell, 1}^{m / q, 2}=c \sqrt{\tau_{s}^{2}-1}\left(Q_{\ell}^{m}\left(\tau_{s}\right) \zeta \sqrt{1-\zeta^{2}} P_{\ell}^{m^{\prime}}(\zeta)\right. \\
& \left.-\tau_{s} Q_{\ell}^{m^{\prime}}\left(\tau_{s}\right) \sqrt{1-\zeta^{2}} P_{\ell}^{m}(\zeta)\right) f_{m}^{q}(\varphi) \\
& f_{\ell, 2}^{m / q, 2}=c\left(\tau_{s} Q_{\ell}^{m}\left(\tau_{s}\right)\left(1-\zeta^{2}\right) P_{\ell}^{m^{\prime}}(\zeta)\right. \\
& \left.+\left(\tau_{s}^{2}-1\right) Q_{\ell}^{m^{\prime}}\left(\tau_{s}\right) \zeta P_{\ell}^{m}(\zeta)\right) \cos \varphi f_{m}^{q}(\varphi) \\
& f_{\ell, 3}^{m / q, 2}=c\left(\tau_{s} Q_{\ell}^{m}\left(\tau_{s}\right)\left(1-\zeta^{2}\right) P_{\ell}^{m^{\prime}}(\zeta)\right. \\
& \left.+\left(\tau_{s}^{2}-1\right) Q_{\ell}^{m^{\prime}}\left(\tau_{s}\right) \zeta P_{\ell}^{m}(\zeta)\right) \sin \varphi f_{m}^{q}(\varphi)
\end{aligned}
$$




$$
\begin{aligned}
& g_{\ell}^{m / q, 2}=-c^{2} M_{\ell, \mathrm{ex}}^{m / q}\left(\mathbf{r}_{0}\right) \sqrt{\tau_{s}^{2}-1} P_{\ell}^{m^{\prime}}\left(\tau_{s}\right) \\
& \cdot \sqrt{1-\zeta^{2}}\left\{\tau_{s}\left[\frac{Q_{\ell}^{m}\left(\tau_{s}\right)}{Q_{\ell}^{m^{\prime}}\left(\tau_{s}\right)}\right] P_{\ell}^{m^{\prime}}(\zeta)-\zeta P_{\ell}^{m}(\zeta)\right\} \\
& \cdot f_{m}^{q}(\varphi)-2 c^{2}\left(\tau_{s}^{2}-\zeta^{2}\right)\left[\widehat{\boldsymbol{\tau}} \times \mathbf{M}_{\ell, \mathrm{ex}}^{m / q}\left(\mathbf{r}_{0}\right) \cdot \widehat{\zeta}\right] P_{\ell}^{m}\left(\tau_{s}\right) \\
& \cdot P_{\ell}^{m}(\zeta) f_{m}^{q}(\varphi) .
\end{aligned}
$$

Finally, the $\kappa=3$ case is structured by the functions

$$
\begin{aligned}
& f_{\ell, 1}^{m / q, 3}=c \sqrt{\tau_{s}^{2}-1} Q_{\ell}^{m}\left(\tau_{s}\right) \zeta P_{\ell}^{m}(\zeta) f_{m}^{q^{\prime}}(\varphi), \\
& f_{\ell, 2}^{m / q, 3}=c \sqrt{1-\zeta^{2}} P_{\ell}^{m}(\zeta)\left(\tau_{s} Q_{\ell}^{m}\left(\tau_{s}\right) \cos \varphi f_{m}^{q^{\prime}}(\varphi)\right. \\
& \left.\quad+\left(\tau_{s}^{2}-1\right) Q_{\ell}^{m^{\prime}}\left(\tau_{s}\right) \sin \varphi f_{m}^{q}(\varphi)\right), \\
& f_{\ell, 3}^{m / q, 3}=c \sqrt{1-\zeta^{2}} P_{\ell}^{m}(\zeta)\left(\tau_{s} Q_{\ell}^{m}\left(\tau_{s}\right) \sin \varphi f_{m}^{q^{\prime}}(\varphi)\right. \\
& \left.\quad-\left(\tau_{s}^{2}-1\right) Q_{\ell}^{m^{\prime}}\left(\tau_{s}\right) \cos \varphi f_{m}^{q}(\varphi)\right), \\
& g_{\ell}^{m / q, 3}=-c^{2} M_{\ell, \mathrm{ex}}^{m / q}\left(\mathbf{r}_{0}\right) \tau_{s} \sqrt{\tau_{s}^{2}-1}\left[\frac{Q_{\ell}^{m}\left(\tau_{s}\right)}{Q_{\ell}^{m^{\prime}}\left(\tau_{s}\right)}\right] P_{\ell}^{m^{\prime}}\left(\tau_{s}\right) \\
& \cdot P_{\ell}^{m}(\zeta) f_{m}^{q^{\prime}}(\varphi)+2 c \sqrt{\tau_{s}^{2}-\zeta^{2}}\left[\widehat{\boldsymbol{\tau}} \times \mathbf{M}_{\ell, \mathrm{ex}}^{m / q}\left(\mathbf{r}_{0}\right) \cdot \widehat{\varphi}\right] \\
& \cdot \sqrt{\tau_{s}^{2}-1} P_{\ell}^{m}\left(\tau_{s}\right) \sqrt{1-\zeta^{2}} P_{\ell}^{m}(\zeta) f_{m}^{q}(\varphi) .
\end{aligned}
$$

All notations, (67)-(69), are valid for any $\tau_{s} \neq \tau_{0}, \zeta \in[-1,1]$, $\varphi \in[0,2 \pi)$, and $\mathbf{r}_{0}=\left(\tau_{0}, \zeta_{0}, \varphi_{0}\right)$, while the unit normal vectors $\widehat{\boldsymbol{\tau}} \equiv \widehat{\boldsymbol{\tau}}\left(\tau_{s}, \zeta, \varphi\right), \widehat{\boldsymbol{\zeta}} \equiv \widehat{\boldsymbol{\zeta}}\left(\tau_{s}, \zeta, \varphi\right)$, and $\widehat{\boldsymbol{\varphi}} \equiv \widehat{\boldsymbol{\varphi}}(\varphi)$ are defined in (36)-(38), the ad hoc function $\rho_{\ell \text {,ex }}^{m / q}\left(\mathbf{r}_{0}\right)$ comes from (47), the dipole source $\mathbf{m}$ is met by (2), and the position vector $\mathbf{r}\left(\tau_{s}, \zeta, \varphi\right)$ is taken from (65), while by definition

$$
\begin{aligned}
& M_{\ell, \mathrm{ex}}^{m / q}\left(\mathbf{r}_{0}\right)=\frac{\mathbf{m}}{4 \pi} \cdot \nabla_{\mathbf{r}_{0}} \rho_{\ell, \mathrm{ex}}^{m / q}\left(\mathbf{r}_{0}\right), \\
& \mathbf{M}_{\ell, \mathrm{ex}}^{m / q}\left(\mathbf{r}_{0}\right)=\frac{\mathbf{m}}{4 \pi} \times \nabla_{\mathbf{r}_{0}} \rho_{\ell, \mathrm{ex}}^{m / q}\left(\mathbf{r}_{0}\right),
\end{aligned}
$$

$$
\text { with } \mathbf{r}_{0}=\left(\tau_{0}, \zeta_{0}, \varphi_{0}\right)
$$

for $\ell \geq 0, m=0,1,2, \ldots, \ell$, and $q=e, o$. Notice that derivations are with respect to the argument. Certainly, relationships (66) accompanied by definitions (67)-(69) are very complicated to accept elaboration with respect to recurrence relations, since in the end a numerical implementation for the resulting systems is inevitable. However, in order to achieve a compact and easy-to-handle analytical formation of these conditions, we expand the already known functions (67)-(69) in terms of the priori orthonormal basis $P_{\ell^{\prime}}^{m^{\prime}} f_{m^{\prime}}^{q^{\prime}}$ for $\ell^{\prime} \geq 0, m^{\prime}=0,1,2, \ldots, \ell^{\prime}$, and $q^{\prime}=e, o$ via

$$
\begin{array}{r}
f_{\ell, j}^{m / q, \kappa}\left(\tau_{s}, \zeta, \varphi\right) \\
=\sum_{\ell^{\prime}=0}^{+\infty} \sum_{m^{\prime}=0}^{\ell^{\prime}} \sum_{q^{\prime}=e, o} \lambda_{\left(\ell, \ell^{\prime}\right), j}^{\left(m, m^{\prime}\right) /\left(q, q^{\prime}\right), \kappa}\left(\tau_{s}\right) P_{\ell^{\prime}}^{m^{\prime}}(\zeta) f_{m^{\prime}}^{q^{\prime}}(\varphi) \\
\text { with } \kappa, j=1,2,3, \\
g_{\ell}^{m / q, \kappa}\left(\tau_{s}, \zeta, \varphi ; \mathbf{r}_{0}\right) \quad \text { with } \kappa=1,2,3
\end{array}
$$

and for every $\ell \geq 0, m=0,1,2, \ldots, \ell$, and $q=e, o$, where, by virtue of the orthogonality properties (44) and (45), we end up with the easily amenable integral representations

$$
\begin{aligned}
& \lambda_{\left(\ell, \ell^{\prime}\right), j}^{\left(m, m^{\prime}\right) /\left(q, q^{\prime}\right), \kappa}\left(\tau_{s}\right)=\frac{2 \ell^{\prime}+1}{4 \pi} \frac{\left(\ell^{\prime}-m^{\prime}\right) !}{\left(\ell^{\prime}+m^{\prime}\right) !} \\
& \cdot \varepsilon_{m^{\prime}} \int_{-1}^{+1} \int_{0}^{2 \pi} f_{\ell, j}^{m / q, \kappa}\left(\tau_{s}, \zeta, \varphi\right) P_{\ell^{\prime}}^{m^{\prime}}(\zeta) f_{m^{\prime}}^{q^{\prime}}(\varphi) d \varphi d \zeta \\
& \mu_{\left(\ell, \ell^{\prime}\right)}^{\left(m, m^{\prime}\right) /\left(q, q^{\prime}\right), \kappa}\left(\tau_{s} ; \mathbf{r}_{0}\right)=\frac{2 \ell^{\prime}+1}{4 \pi} \frac{\left(\ell^{\prime}-m^{\prime}\right) !}{\left(\ell^{\prime}+m^{\prime}\right) !} \\
& \cdot \varepsilon_{m^{\prime}} \int_{-1}^{+1} \int_{0}^{2 \pi} g_{\ell}^{m / q, \kappa}\left(\tau_{s}, \zeta, \varphi ; \mathbf{r}_{0}\right) P_{\ell^{\prime}}^{m^{\prime}}(\zeta) \\
& \cdot f_{m^{\prime}}^{q^{\prime}}(\varphi) d \varphi d \zeta
\end{aligned}
$$

for every $\left(\ell, \ell^{\prime}\right) \geq 0,\left(m, m^{\prime}\right)=0,1,2, \ldots,\left(\ell, \ell^{\prime}\right)$, and $\left(q, q^{\prime}\right)=$ $e, o$ with $\kappa, j=1,2,3$. The last step includes supersession of expressions (71), provided (72), into (66) and processing of a handy orthogonal argumentation based on (44) and (45), so as to conclude to

$$
\sum_{\ell=0}^{+\infty} \sum_{m=0}^{\ell} \sum_{q=e, o}\left[\sum_{j=1}^{3}\left(\lambda_{\left(\ell, \ell^{\prime}\right), j}^{\left(m, m^{\prime}\right) /\left(q, q^{\prime}\right), \kappa}\left(\tau_{s}\right) b_{\ell, j}^{m / q}\right)-\mu_{\left(\ell, \ell^{\prime}\right)}^{\left(m, m^{\prime}\right) /\left(q, q^{\prime}\right), \kappa}\left(\tau_{s} ; \mathbf{r}_{0}\right)\right]=0 \quad \text { for } \mathbf{r}_{0}=\left(\tau_{0}, \zeta_{0}, \varphi_{0}\right)
$$

while $\kappa=1,2,3$, as well as $\ell^{\prime} \geq 0, m^{\prime}=0,1,2, \ldots, \ell^{\prime}$, and $q^{\prime}=e, o$, offer all existing combinations regarding
(73). In doing so, we achieved conveying the arisen difficulty from the boundary conditions (66) to the simple calculation 
of integrals (72), which just incorporate trigonometric and associated Legendre functions, so as to become a matter of performing trivial integrations or applying a standard computational code. Thereafter, (73) represents systems of linear algebraic equations, which can be solved via usual cutoff techniques by the imposition of an indispensable common upper limit for both the degree indexes; let us assume $\ell=\ell^{\prime}=$ $0,1,2, \ldots, L$, in order to obtain quadrature forms, such as

$$
\begin{aligned}
\mathbb{A}_{L}\left(\tau_{s}\right) \mathbf{x}_{L} & =\mathbf{z}_{L}\left(\tau_{s} ; \mathbf{r}_{0}\right) \Longrightarrow \\
\mathbf{x}_{L} & =\mathbb{A}_{L}^{-1}\left(\tau_{s}\right) \mathbf{z}_{L}\left(\tau_{s} ; \mathbf{r}_{0}\right) \\
& \text { for } \mathbf{r}_{0}=\left(\tau_{0}, \zeta_{0}, \varphi_{0}\right),
\end{aligned}
$$

where for $m=m^{\prime}=0,1,2, \ldots, \ell$ and $q=q^{\prime}=e, o$, as well as $\kappa, j=1,2,3$, we have

$$
\mathbb{A}_{L}\left(\tau_{s}\right)=\left[\begin{array}{ccc}
\ddots & \vdots & \cdot \\
\cdots & \lambda_{(\ell, \ell), j}^{(m, m) /(q, q), \kappa}\left(\tau_{s}\right) & \cdots \\
. & \vdots & \ddots
\end{array}\right],
$$

$$
\begin{aligned}
\mathbf{x}_{L} & =\left[\begin{array}{c}
\vdots \\
b_{\ell, j}^{m / q} \\
\vdots
\end{array}\right], \\
\mathbf{z}_{L}\left(\tau_{s} ; \mathbf{r}_{0}\right) & =\left[\begin{array}{c}
\vdots \\
\mu_{(\ell, \ell), j}^{(m, m) /(q, q), \kappa}\left(\tau_{s} ; \mathbf{r}_{0}\right) \\
\vdots
\end{array}\right],
\end{aligned}
$$

which denote the $3(L+1) \times m \times q$ squared-type invertible matrix of the coefficients of the unknowns, the vector of the unknown coefficients, and the vector of the known constants, respectively. The vector $\mathbf{x}_{L}$ specifies the system's solution through (74), being able to evaluate the unknown constant coefficients $\mathbf{b}_{\ell, \text { ex }}^{m / q}=b_{\ell, 1}^{m / q} \widehat{\mathbf{x}}_{1}+b_{\ell, 2}^{m / q} \widehat{\mathbf{x}}_{2}+b_{\ell, 3}^{m / q} \widehat{\mathbf{x}}_{3}$ for every value $\ell=0,1,2, \ldots, L, m=0,1,2, \ldots, \ell$, and $q=e, o$ up to a certain level, where the desired accuracy is achieved. Thus, by substitution of (62) and (63) into (59), we reach the analytical form of the scattered magnetic field for this occasion, that is,

$$
\mathbf{H}_{2}^{s}(\mathbf{r})=\sum_{\ell=0}^{+\infty} \sum_{m=0}^{\ell} \sum_{q=e, o}\left\{\left[\mathbf{b}_{\ell, \mathrm{ex}}^{m / q}+\mathbf{r}\left(\frac{\mathbf{m}}{4 \pi} \cdot \nabla_{\mathbf{r}_{0}} \rho_{\ell, \mathrm{ex}}^{m / q}\left(\mathbf{r}_{0}\right)\right) \frac{P_{\ell}^{m^{\prime}}\left(\tau_{s}\right)}{Q_{\ell}^{m^{\prime}}\left(\tau_{s}\right)}\right] Q_{\ell}^{m}(\tau) P_{\ell}^{m}(\zeta) f_{m}^{q}(\varphi)\right\}
$$

for $\mathbf{r} \in \Omega$, satisfying (61), wherein, upon solution (74), the prolate spheroidal modes, indicated by (64) and (65), can be readily embodied.

The simplest case of this project concerns the thirdorder scattered magnetic field at $n=3$ due to the constant character of the corresponding incident field (9). Similarly to the Rayleigh approximation, here we are obliged to solve the potential boundary value problem (32) with the Neumann boundary condition (24) for $n=3$, that is,

$$
\mathbf{H}_{3}^{s}(\mathbf{r})=\nabla \Phi_{3}^{s}(\mathbf{r}) \quad \text { with } \Delta \Phi_{3}^{s}(\mathbf{r})=0 \text { for } \mathbf{r} \in \Omega, \text { where } \widehat{\boldsymbol{\tau}}\left(\mathbf{r}_{s}\right) \cdot\left[\mathbf{H}_{3}^{p}\left(\mathbf{r}_{s}\right)+\mathbf{H}_{3}^{s}\left(\mathbf{r}_{s}\right)\right]=0
$$

by means of $\mathbf{r}_{s}=\left(\tau_{s}, \zeta, \varphi\right)$, where $\widehat{\boldsymbol{\tau}}$ is given through (36) for $\tau=\tau_{s}$. In order to comply with the demand of well-behaved solutions at infinity via (26), we again choose

$$
\mathbf{H}_{3}^{s}(\mathbf{r})=\sum_{\ell=0}^{+\infty} \sum_{m=0}^{\ell} \sum_{q=e, o} c_{\ell, \mathrm{ex}}^{m / q} \nabla\left[Q_{\ell}^{m}(\tau) P_{\ell}^{m}(\zeta) f_{m}^{q}(\varphi)\right]
$$

whereas $c_{\ell, \text { ex }}^{m / q}$ for $\ell \geq 0, m=0,1,2, \ldots, \ell$, and $q=e, o$ stand for the constant coefficients to be determined from (77). Consequently, invoking the source (9) and projecting $\mathbf{m}$ from (2), then, accordingly to (78), the convenient relation (49) is applied to (77) in order to receive

for every $\mathbf{r} \in \Omega$,

$$
\begin{aligned}
& \left\{-c_{0, \mathrm{ex}}^{0 / e}+\sum_{\ell=1}^{\infty} \sum_{m=0}^{\ell} \sum_{q=e, o} \frac{c_{\ell, \mathrm{ex}}^{m / q} f_{m}^{q}(\varphi)}{(2 \ell+1)}\left[\ell(\ell-m+1) Q_{\ell+1}^{m}\left(\tau_{s}\right)-(\ell+1)(\ell+m) Q_{\ell-1}^{m}\left(\tau_{s}\right)\right] P_{\ell}^{m}(\zeta)-\frac{c \sqrt{\tau_{s}^{2}-1}}{\pi}\left(m_{1} \zeta \sqrt{\tau_{s}^{2}-1}\right.\right. \\
& \left.\left.\quad+m_{2} \tau_{s} \sqrt{1-\zeta^{2}} \cos \varphi+m_{3} \tau_{s} \sqrt{1-\zeta^{2}} \sin \varphi\right)\right\}=0
\end{aligned}
$$


for every $\zeta \in[-1,1]$ and $\varphi \in[0,2 \pi)$. Since within (79) we can write $1=P_{0}^{0}(\zeta), \zeta=P_{1}^{0}(\zeta)$, and $\sqrt{1-\zeta^{2}}=P_{1}^{1}(\zeta)$, obviously standard orthogonality arguments in terms of (44) and (45) yield

$$
c_{0, \mathrm{ex}}^{0 / e}=0, \quad c_{\ell, \mathrm{ex}}^{m / q}=0,
$$

$$
\text { for every } \ell \geq 2, m=0,1,2, \ldots, \ell, q=e, o \text {, }
$$

while the remainder ones read

$$
\begin{aligned}
& c_{1, \mathrm{ex}}^{0 / e}=\frac{c m_{1}}{\pi} \frac{1}{Q_{1}^{\prime}\left(\tau_{s}\right)}, \\
& c_{1, \mathrm{ex}}^{1 / e}=\frac{c m_{2}}{\pi} \frac{\tau_{s}}{\sqrt{\tau_{s}^{2}-1} Q_{1}^{1^{\prime}}\left(\tau_{s}\right)}, \\
& c_{1, \mathrm{ex}}^{1 / o}=\frac{c m_{3}}{\pi} \frac{\tau_{s}}{\sqrt{\tau_{s}^{2}-1} Q_{1}^{1^{\prime}}\left(\tau_{s}\right)},
\end{aligned}
$$

once we enforce the useful identity

$$
\begin{aligned}
&(2 \ell+1)\left(\tau_{s}^{2}-1\right) Q_{\ell}^{m^{\prime}}\left(\tau_{s}\right) \\
&= \ell(\ell-m+1) Q_{\ell+1}^{m}\left(\tau_{s}\right) \\
&-(\ell+1)(\ell+m) Q_{\ell-1}^{m}\left(\tau_{s}\right),
\end{aligned}
$$

prime denoting derivation with respect to $\tau_{s}$. Inserting (80) and (81) into (78), we get

$$
\begin{gathered}
\mathbf{H}_{3}^{\mathcal{s}}(\mathbf{r})=\frac{\mathbf{m}}{\pi} \\
\quad\left\{\frac{\sqrt{\tau^{2}-1}}{\sqrt{\tau^{2}-\zeta^{2}}}\left[\frac{\partial \mathbf{f}(\tau)}{\partial \tau} \zeta+\frac{\partial \mathbf{g}(\tau, \varphi)}{\partial \tau} \sqrt{1-\zeta^{2}}\right] \otimes \widehat{\boldsymbol{\tau}}\right. \\
+\frac{1}{\sqrt{\tau^{2}-\zeta^{2}}}\left[-\mathbf{f}(\tau) \sqrt{1-\zeta^{2}}+\mathbf{g}(\tau, \varphi) \zeta\right] \otimes \widehat{\zeta} \\
\left.+\frac{1}{\sqrt{\tau^{2}-1}} \frac{\partial \mathbf{g}(\tau, \varphi)}{\partial \varphi} \otimes \widehat{\boldsymbol{\varphi}}\right\} \quad \text { for } \mathbf{r} \in \Omega,
\end{gathered}
$$

which introduces a compact dyadic formation, bearing in mind that

$$
\begin{aligned}
\mathbf{f}(\tau) & =\widehat{\mathbf{x}}_{1} \frac{Q_{1}(\tau)}{Q_{1}^{\prime}\left(\tau_{s}\right)}, \\
\mathbf{g}(\tau, \varphi) & =\left(\widehat{\mathbf{x}}_{2} \cos \varphi+\widehat{\mathbf{x}}_{3} \sin \varphi\right) \frac{\tau_{s}}{\sqrt{\tau_{s}^{2}-1}} \frac{Q_{1}^{1}(\tau)}{Q_{1}^{1^{\prime}}\left(\tau_{s}\right)}
\end{aligned}
$$

are conveniently chosen functions for every $\tau \in\left[\tau_{s},+\infty\right)$ and $\varphi \in[0,2 \pi)$.

3.3. The $\mathbf{E}_{1}^{s}$ and $\mathbf{E}_{3}^{s}$ Electric Fields. The first nonvanishing electric term appears as $n=1$ at the low-frequency regime and it is interconnected with $\mathbf{H}_{2}^{s}$ via (30). Hence, we incorporate the latter with the already known field (76) and with aid of a simple differential identity we obtain

$$
\mathbf{E}_{1}^{s}(\mathbf{r})=-\frac{1}{2} \sqrt{\frac{\mu}{\varepsilon}} \sum_{\ell=0}^{+\infty} \sum_{m=0}^{\ell} \sum_{q=e, o}\left\{\nabla u_{\ell, \mathrm{ex}}^{m / q}(\mathbf{r}) \times\left[\mathbf{b}_{\ell, \mathrm{ex}}^{m / q}+\mathbf{r}\left(\frac{\mathbf{m}}{4 \pi} \cdot \nabla_{\mathbf{r}_{0}} \rho_{\ell, \mathrm{ex}}^{m / q}\left(\mathbf{r}_{0}\right)\right) \frac{P_{\ell}^{m^{\prime}}\left(\tau_{s}\right)}{Q_{\ell}^{m^{\prime}}\left(\tau_{s}\right)}\right]\right\} \quad \text { for } \mathbf{r} \in \Omega
$$

since $\nabla \times \mathbf{b}_{\ell, \mathrm{ex}}^{m / q}=\mathbf{0}$ and $\nabla \times \mathbf{r}=\mathbf{0}$, where $u_{\ell, \mathrm{ex}}^{m / q}$ is the right-hand side expression of (41), while $\nabla u_{\ell, \mathrm{ex}}^{m / q}$ is taken from (49), the singular quantity $\rho_{\ell, \mathrm{ex}}^{m / q}\left(\mathbf{r}_{0}\right)$ follows from (47), and, finally, the constant coefficients $\mathbf{b}_{\ell, \text { ex }}^{m / q}$ are provided via the $n=2$ magnetic situation, all written in view of $\ell \geq 0, m=0,1,2, \ldots, \ell$, and $q=e, o$.

Our final task is the calculation of the exact low-frequency problem at $n=3$, where here the main difficulty is due to the integral implication with the electric field (85) of the offered solution; see (31) for instance. Henceforth, we deal with a boundary value problem, being the outcome of the combination of (31) and of the second part of (24) for $n=3$, that is,

$$
\mathbf{E}_{3}^{s}(\mathbf{r})=\mathbf{X}_{3}^{s}(\mathbf{r})-\frac{3}{2 \pi} \iiint_{\Omega} \frac{\mathbf{E}_{1}^{s}\left(\mathbf{r}^{\prime}\right)}{\left|\mathbf{r}-\mathbf{r}^{\prime}\right|} d \Omega^{\prime}
$$

with $\Delta \mathbf{X}_{3}^{s}(\mathbf{r})=\mathbf{0}$, where $\widehat{\boldsymbol{\tau}}\left(\mathbf{r}_{s}\right) \times\left[\mathbf{E}_{3}^{p}\left(\mathbf{r}_{s}\right)+\mathbf{E}_{3}^{s}\left(\mathbf{r}_{s}\right)\right]=\mathbf{0}$ for every $\mathbf{r} \in \Omega$ and on $\mathbf{r}_{s}=\left(\tau_{s}, \zeta, \varphi\right)$ with $\zeta \in[-1,1]$ and $\varphi \in[0,2 \pi)$, while we recall the corresponding incident electric field (11). Field (86) is solenoidal, which is secured via

$$
\begin{aligned}
& \nabla \cdot \mathbf{X}_{3}^{s}(\mathbf{r})+\nabla \cdot\left[-\frac{3}{2 \pi} \iiint_{\Omega} \frac{\mathbf{E}_{1}^{s}\left(\mathbf{r}^{\prime}\right)}{\left|\mathbf{r}-\mathbf{r}^{\prime}\right|} d \Omega^{\prime}\right]=0 \Longrightarrow \\
& \nabla \cdot \mathbf{X}_{3}^{s}(\mathbf{r})-\frac{3}{2 \pi} \iiint_{\Omega} \nabla \frac{1}{\left|\mathbf{r}-\mathbf{r}^{\prime}\right|} \cdot \mathbf{E}_{1}^{s}\left(\mathbf{r}^{\prime}\right) d \Omega^{\prime}=0,
\end{aligned}
$$

since $\nabla \cdot \mathbf{E}_{1}^{\mathcal{s}}\left(\mathbf{r}^{\prime}\right)=0$, whereas (12) can also be employed. Obviously $\mathbf{E}_{3}^{s}$ is a vector field, retaining three components, which require three scalar boundary conditions. However, we notice that (86) offers two of them, forcing us to retrieve the third one by the application of the restriction condition (87) on the surface of the metallic prolate spheroid, meaning 


$$
\begin{aligned}
\nabla \cdot\left[\mathbf{X}_{3}^{s}(\mathbf{r})-\frac{3}{2 \pi} \iiint_{\Omega} \frac{\mathbf{E}_{1}^{s}\left(\mathbf{r}^{\prime}\right)}{\left|\mathbf{r}-\mathbf{r}^{\prime}\right|} d \Omega^{\prime}\right] & =0 \\
& \text { for } \mathbf{r}_{s}=\left(\tau_{s}, \zeta, \varphi\right),
\end{aligned}
$$

which completes the well-posed analysis. In order for it to be compatible with the radiation condition (26) for $\mathbf{E}_{3}^{s}$, we expand $\mathbf{X}_{3}^{s}$ as usual, being an exterior potential,

$$
\mathbf{X}_{3}^{s}(\mathbf{r})=\sum_{\ell=0}^{+\infty} \sum_{m=0}^{\ell} \sum_{q=e, o} \mathbf{d}_{\ell, \mathrm{ex}}^{m / q}\left[Q_{\ell}^{m}(\tau) P_{\ell}^{m}(\zeta) f_{m}^{q}(\varphi)\right]
$$

for every $\mathbf{r} \in \Omega$,

where, similarly to (64), we have

$$
\begin{aligned}
& \mathbf{d}_{\ell, \mathrm{ex}}^{m / q}=\sum_{j=1}^{3} d_{\ell, j}^{m / q} \widehat{\mathbf{x}}_{j}=d_{\ell, 1}^{m / q} \widehat{\mathbf{x}}_{1}+d_{\ell, 2}^{m / q} \widehat{\mathbf{x}}_{2}+d_{\ell, 3}^{m / q} \widehat{\mathbf{x}}_{3}=\left(\tau^{2}\right. \\
& \left.-\zeta^{2}\right)^{-1 / 2}\left\{\left(d_{\ell, 1}^{m / q} \zeta \sqrt{\tau^{2}-1}+d_{\ell, 2}^{m / q} \tau \sqrt{1-\zeta^{2}} \cos \varphi\right.\right. \\
& \left.+d_{\ell, 3}^{m / q} \tau \sqrt{1-\zeta^{2}} \sin \varphi\right) \widehat{\boldsymbol{\tau}}+\left(-d_{\ell, 1}^{m / q} \tau \sqrt{1-\zeta^{2}}\right. \\
& \left.\left.+d_{\ell, 2}^{m / q} \zeta \sqrt{\tau^{2}-1} \cos \varphi+d_{\ell, 3}^{m / q} \zeta \sqrt{\tau^{2}-1} \sin \varphi\right) \widehat{\zeta}\right\} \\
& +\left(-d_{\ell, 2}^{m / q} \sin \varphi+d_{\ell, 3}^{m / q} \cos \varphi\right) \widehat{\boldsymbol{\varphi}} \\
& \quad \text { for } \ell \geq 0, m=0,1,2, \ldots, \ell, q=e, o .
\end{aligned}
$$

In the prolate spheroidal geometry, we utilize the infinitesimal elementary volume

$$
\begin{aligned}
d \Omega^{\prime}= & c^{3}\left(\tau^{\prime 2}-\zeta^{\prime 2}\right) d \tau^{\prime} d \zeta^{\prime} d \varphi^{\prime} \\
& \text { for } \tau^{\prime} \in\left[\tau_{s},+\infty\right), \zeta^{\prime} \in[-1,1], \varphi^{\prime} \in[0,2 \pi),
\end{aligned}
$$

to rewrite the particular integral solution that appears into (86) for any $\mathbf{r} \neq \mathbf{r}_{0}$ as

$$
\begin{aligned}
& -\frac{3}{2 \pi} \iiint_{\Omega} \frac{\mathbf{E}_{1}^{s}\left(\mathbf{r}^{\prime}\right)}{\left|\mathbf{r}-\mathbf{r}^{\prime}\right|} d \Omega^{\prime}=-\frac{3 c^{3}}{2 \pi} \\
& \quad \int_{0}^{2 \pi} \int_{-1}^{+1} \int_{\tau_{s}}^{+\infty} \frac{\mathbf{E}_{1}^{s}\left(\mathbf{r}^{\prime}\right)}{\left|\mathbf{r}-\mathbf{r}^{\prime}\right|}\left(\tau^{\prime 2}-\zeta^{\prime 2}\right) d \tau^{\prime} d \zeta^{\prime} d \varphi^{\prime} \\
& \quad=-\frac{3 c^{3}}{2 \pi}\left\{\lim _{e \rightarrow 0} \int_{0}^{2 \pi} \int_{-1}^{+1} \int_{\tau_{s}}^{\tau_{0}-e} \frac{\mathbf{E}_{1}^{s}\left(\mathbf{r}^{\prime}\right)}{\left|\mathbf{r}-\mathbf{r}^{\prime}\right|}\left(\tau^{\prime 2}-\zeta^{\prime 2}\right) d \tau^{\prime} d \zeta^{\prime} d \varphi^{\prime}\right. \\
& +\lim _{e \rightarrow 0} \int_{0}^{2 \pi} \int_{-1}^{+1} \int_{\tau_{0}-e}^{\tau_{0}+e} \frac{\mathbf{E}_{1}^{s}\left(\mathbf{r}^{\prime}\right)}{\left|\mathbf{r}-\mathbf{r}^{\prime}\right|}\left(\tau^{\prime 2}-\zeta^{\prime 2}\right) d \tau^{\prime} d \zeta^{\prime} d \varphi^{\prime} \\
& \left.\quad+\lim _{e \rightarrow 0} \int_{0}^{2 \pi} \int_{-1}^{+1} \int_{\tau_{0}+e}^{+\infty} \frac{\mathbf{E}_{1}^{s}\left(\mathbf{r}^{\prime}\right)}{\left|\mathbf{r}-\mathbf{r}^{\prime}\right|}\left(\tau^{\prime 2}-\zeta^{\prime 2}\right) d \tau^{\prime} d \zeta^{\prime} d \varphi^{\prime}\right\}
\end{aligned}
$$$$
\text { for } \mathbf{r} \in \Omega
$$

and $\mathbf{r}^{\prime}=\left(\tau^{\prime}, \zeta^{\prime}, \varphi^{\prime}\right)$, whereas we have implied a specific technique to bound the singular point, by introducing a very small positive number $0<e \ll 1$, while for reasons of simplicity and without loss of the generality, we assume that $\left(\zeta^{\prime}, \varphi^{\prime}\right) \neq\left(\zeta_{0}, \varphi_{0}\right)$ by definition. The first and the third integral of (92) are analytic, since they exclude the singular point; hence therein we can use collectively (46) to represent the different areas, by applying the lower branch for $\tau^{\prime}<\tau_{0}-e$ and the upper one for $\tau^{\prime}>\tau_{0}+e$, respectively. This action conducts to

$$
\begin{aligned}
& -\frac{3 c^{3}}{2 \pi} \lim _{e \rightarrow 0} \int_{0}^{2 \pi} \int_{-1}^{+1} \int_{\tau_{s}}^{\tau_{0}-e} \frac{\mathbf{E}_{1}^{s}\left(\mathbf{r}^{\prime}\right)}{\left|\mathbf{r}-\mathbf{r}^{\prime}\right|}\left(\tau^{\prime 2}-\zeta^{\prime 2}\right) d \tau^{\prime} d \zeta^{\prime} d \varphi^{\prime} \\
& =\sum_{\ell=0}^{+\infty} \sum_{m=0}^{\ell} \sum_{q=e, o} \mathbf{s}_{\ell, \mathrm{in}}^{m / q} u_{\ell, \mathrm{in}}^{m / q}(\mathbf{r}) \quad \text { for } \mathbf{r} \in \Omega, \\
& -\frac{3 c^{3}}{2 \pi} \lim _{e \rightarrow 0} \int_{0}^{2 \pi} \int_{-1}^{+1} \int_{\tau_{0}+e}^{+\infty} \frac{\mathbf{E}_{1}^{s}\left(\mathbf{r}^{\prime}\right)}{\left|\mathbf{r}-\mathbf{r}^{\prime}\right|}\left(\tau^{\prime 2}-\zeta^{\prime 2}\right) d \tau^{\prime} d \zeta^{\prime} d \varphi^{\prime} \\
& =\sum_{\ell=0}^{+\infty} \sum_{m=0}^{\ell} \sum_{q=e, o} \mathbf{s}_{\ell, \mathrm{ex}}^{m / q} u_{\ell, \mathrm{ex}}^{m / q}(\mathbf{r}) \quad \text { for } \mathbf{r} \in \Omega,
\end{aligned}
$$

which are harmonic, where the leading constants for $\ell \geq 0$, $m=0,1,2, \ldots, \ell$, and $q=e, o$,

$$
\begin{aligned}
\mathbf{s}_{\ell, \mathrm{in}}^{m / q} & =-\frac{3 c^{3}}{2 \pi} \lim _{e \rightarrow 0} \int_{0}^{2 \pi} \int_{-1}^{+1} \int_{\tau_{s}}^{\tau_{0}-e}\left(\tau^{\prime 2}-\zeta^{\prime 2}\right) \rho_{\ell, \mathrm{ex}}^{m / q}\left(\mathbf{r}^{\prime}\right) \\
\cdot & \mathbf{E}_{1}^{s}\left(\mathbf{r}^{\prime}\right) d \tau^{\prime} d \zeta^{\prime} d \varphi^{\prime}, \\
\mathbf{s}_{\ell, \mathrm{ex}}^{m / q} & =-\frac{3 c^{3}}{2 \pi} \lim _{e \rightarrow 0} \int_{0}^{2 \pi} \int_{-1}^{+1} \int_{\tau_{0}+e}^{+\infty}\left(\tau^{\prime 2}-\zeta^{\prime 2}\right) \rho_{\ell, \mathrm{in}}^{m / q}\left(\mathbf{r}^{\prime}\right) \\
\cdot & \mathbf{E}_{1}^{s}\left(\mathbf{r}^{\prime}\right) d \tau^{\prime} d \zeta^{\prime} d \varphi^{\prime},
\end{aligned}
$$

respectively, are written by virtue of (47) at the relative integration interval on $\mathbf{r}^{\prime}=\left(\tau^{\prime}, \zeta^{\prime}, \varphi^{\prime}\right)$. On the other hand, this is not the issue with the second integral, which contains the singularity and implying (91), it provides us with the particular solution in (31) via

$$
\begin{aligned}
& \Delta\left\{-\frac{3}{2 \pi} \lim _{e \rightarrow 0} \int_{0}^{2 \pi} \int_{-1}^{+1} \int_{\tau_{0}-e}^{\tau_{0}+e} \frac{\mathbf{E}_{1}^{s}\left(\mathbf{r}^{\prime}\right)}{\left|\mathbf{r}-\mathbf{r}^{\prime}\right|} d \Omega^{\prime}\right\} \\
& =6 \lim _{e \rightarrow 0} \int_{0}^{2 \pi} \int_{-1}^{+1} \int_{\tau_{0}-e}^{\tau_{0}+e} \delta\left(\mathbf{r}-\mathbf{r}^{\prime}\right) \mathbf{E}_{1}^{s}\left(\mathbf{r}^{\prime}\right) d \Omega^{\prime} \\
& =6 \mathbf{E}_{1}^{s}(\mathbf{r}) \text { for } \mathbf{r} \in \Omega
\end{aligned}
$$

in terms of the Dirac function $\delta\left(\mathbf{r}-\mathbf{r}^{\prime}\right)$ in Laplace's fundamental solution [14]. Yet, this term is treated differently, by expanding it with respect to the orthonormal basis $P_{\ell}^{m} f_{m}^{q}$,

$$
\begin{gathered}
-\frac{3 c^{3}}{2 \pi} \lim _{\varepsilon \rightarrow 0} \int_{0}^{2 \pi} \int_{-1}^{+1} \int_{\tau_{0}-\varepsilon}^{\tau_{0}+\varepsilon} \frac{\mathbf{E}_{1}^{s}\left(\mathbf{r}^{\prime}\right)}{\left|\mathbf{r}-\mathbf{r}^{\prime}\right|}\left(\tau^{\prime 2}-\zeta^{\prime 2}\right) d \tau^{\prime} d \zeta^{\prime} d \varphi^{\prime} \\
=\sum_{\ell=0}^{+\infty} \sum_{m=0}^{\ell} \sum_{q=e, o} \mathbf{S}_{\ell}^{m / q}(\tau) P_{\ell}^{m}(\zeta) f_{m}^{q}(\varphi)
\end{gathered}
$$


for $\mathbf{r} \in \Omega$, where, taking into account (91) and the inner products (44) and (45), we have

$$
\begin{aligned}
& \mathbf{S}_{\ell}^{m / q}(\tau)=-\frac{3(2 \ell+1)}{8 \pi^{2}} \frac{(\ell-m) !}{(\ell+m) !} \\
& \cdot \varepsilon_{m} \int_{-1}^{+1} \int_{0}^{2 \pi}\left\{\lim _{e \rightarrow 0} \int_{0}^{2 \pi} \int_{-1}^{+1} \int_{\tau_{0}-e}^{\tau_{0}+e} \frac{\mathbf{E}_{1}^{s}\left(\mathbf{r}^{\prime}\right)}{\left|\mathbf{r}-\mathbf{r}^{\prime}\right|} d \Omega^{\prime}\right\} \\
& \cdot P_{\ell}^{m}(\zeta) f_{m}^{q}(\varphi) d \varphi d \zeta
\end{aligned}
$$

for $\tau \geq \tau_{s}$. Gathering all information in between (92) and (98), integral (92) is rewritten as

$$
\begin{aligned}
& -\frac{3}{2 \pi} \iiint_{\Omega} \frac{\mathbf{E}_{1}^{s}\left(\mathbf{r}^{\prime}\right)}{\left|\mathbf{r}-\mathbf{r}^{\prime}\right|} d \Omega^{\prime} \\
& \quad=\sum_{\ell=0}^{+\infty} \sum_{m=0}^{\ell} \sum_{q=e, o}\left[\mathbf{s}_{\ell, \mathrm{in}}^{m / q} P_{\ell}^{m}(\tau)+\mathbf{s}_{\ell, \mathrm{ex}}^{m / q} Q_{\ell}^{m}(\tau)+\mathbf{S}_{\ell}^{m / q}(\tau)\right] \\
& \quad \cdot P_{\ell}^{m}(\zeta) f_{m}^{q}(\varphi)
\end{aligned}
$$

for every $\mathbf{r} \in \Omega$, which combined with (89) yields the scattered field (86). Under the aim of expanding the incident field (11) in the same manner, we incorporate (46) and (47) for $\mathbf{r}_{s}<\mathbf{r}_{0}$, which is in fact the surface of the body, whereas $\mathbf{E}_{3}^{p}$ is activated and since $\mathbf{R}=\mathbf{r}-\mathbf{r}_{0}$, then

$$
\begin{array}{r}
\mathbf{E}_{3}^{p}\left(\mathbf{r} ; \mathbf{r}_{0}\right) \\
=\sum_{\ell=0}^{+\infty} \sum_{m=0}^{\ell} \sum_{q=e, o} \mathbf{I}_{\ell, \mathrm{ex}}^{m / q}\left(\tau, \zeta, \varphi ; \mathbf{r}_{0}\right) P_{\ell}^{m}(\tau) P_{\ell}^{m}(\zeta) f_{m}^{q}(\varphi) \\
\text { for } \mathbf{r}<\mathbf{r}_{0},
\end{array}
$$

where

$$
\begin{aligned}
& \mathbf{I}_{\ell, \mathrm{ex}}^{m / q}\left(\tau, \zeta, \varphi ; \mathbf{r}_{0}\right)=-\frac{3}{4 \pi} \sqrt{\frac{\mu}{\varepsilon}} \mathbf{m} \\
& \times\left[\mathbf{r}(\tau, \zeta, \varphi)-\mathbf{r}_{0}\right] \rho_{\ell, \mathrm{ex}}^{m / q}\left(\mathbf{r}_{0}\right) \\
& \text { with } \mathbf{r}_{0}=\left(\tau_{0}, \zeta_{0}, \varphi_{0}\right)
\end{aligned}
$$

at $\mathbf{r} \in \Omega$. We are ready to precede fitting of the above analysis to (86) and (88), which are the boundary conditions to be satisfied. From this point forward, we deal with the problem in a very similar way to that for obtaining $\mathbf{H}_{2}^{s}$, since it possess the same complexity. Hence, putting all the mathematical tools together, (86) and (88) with (89)-(101) merge to

$$
\begin{aligned}
& \sum_{\ell=0}^{+\infty} \sum_{m=0}^{\ell} \sum_{q=e, o}\left[\sum_{j=1}^{3} \vec{f}_{\ell, j}^{m / q, \kappa}\left(\tau_{s}, \zeta, \varphi\right) d_{\ell, j}^{m / q}-\vec{g}_{\ell}^{m / q, \kappa}\left(\tau_{s}, \zeta, \varphi ; \mathbf{r}_{0}\right)\right] \\
& =0 \quad \text { for any } \kappa=1,2,3,
\end{aligned}
$$

where, for $\mathbf{r}_{0}=\left(\rho_{0}, \theta_{0}, \varphi_{0}\right)$, the $\kappa=1$ case, originated from reinforcement (88), constitutes

$$
\begin{aligned}
& \bar{f}_{\ell, 1}^{m / q, 1}=\frac{1}{c\left(\tau_{s}^{2}-\zeta^{2}\right)}\left[\left(\tau_{s}^{2}-1\right) Q_{\ell}^{m^{\prime}}\left(\tau_{s}\right) \zeta P_{\ell}^{m}(\zeta)\right. \\
& \left.+\tau_{s} Q_{\ell}^{m}\left(\tau_{s}\right)\left(1-\zeta^{2}\right) P_{\ell}^{m^{\prime}}(\zeta)\right] f_{m}^{q}(\varphi), \\
& \bar{f}_{\ell, 2}^{m / q, 1}=\left[\frac { \sqrt { \tau _ { s } ^ { 2 } - 1 } \sqrt { 1 - \zeta ^ { 2 } } } { c ( \tau _ { s } ^ { 2 } - \zeta ^ { 2 } ) } \left(\tau_{s} Q_{\ell}^{m^{\prime}}\left(\tau_{s}\right) P_{\ell}^{m}(\zeta)\right.\right. \\
& \left.-Q_{\ell}^{m}\left(\tau_{s}\right) \zeta P_{\ell}^{m^{\prime}}(\zeta)\right) \cos \varphi f_{m}^{q}(\varphi) \\
& \left.-\frac{1}{c \sqrt{\tau_{s}^{2}-1} \sqrt{1-\zeta^{2}}} Q_{\ell}^{m}\left(\tau_{s}\right) P_{\ell}^{m}(\zeta) \sin \varphi f_{m}^{q^{\prime}}(\varphi)\right] \\
& \bar{f}_{\ell, 3}^{m / q, 1}=\left[\frac { \sqrt { \tau _ { s } ^ { 2 } - 1 } \sqrt { 1 - \zeta ^ { 2 } } } { c ( \tau _ { s } ^ { 2 } - \zeta ^ { 2 } ) } \left(\tau_{s} Q_{\ell}^{m^{\prime}}\left(\tau_{s}\right) P_{\ell}^{m}(\zeta)\right.\right. \\
& \left.-Q_{\ell}^{m}\left(\tau_{s}\right) \zeta P_{\ell}^{m^{\prime}}(\zeta)\right) \sin \varphi f_{m}^{q}(\varphi) \\
& \left.+\frac{1}{c \sqrt{\tau_{s}^{2}-1} \sqrt{1-\zeta^{2}}} Q_{\ell}^{m}\left(\tau_{s}\right) P_{\ell}^{m}(\zeta) \cos \varphi f_{m}^{q^{\prime}}(\varphi)\right] \\
& \bar{g}_{\ell}^{m / q, 1}=-\sqrt{\tau_{s}^{2}-1}\left(c \sqrt{\tau_{s}^{2}-\zeta^{2}}\right)^{-1} \widehat{\boldsymbol{\tau}} \cdot\left\{\mathbf{S}_{\ell}^{m / q^{\prime}}\left(\tau_{s}\right)\right. \\
& \left.-\left[\frac{\mathbf{s}_{\ell}^{m / q}\left(\tau_{s}\right)}{P_{\ell}^{m}\left(\tau_{s}\right)}\right] P_{\ell}^{m^{\prime}}\left(\tau_{s}\right)\right\} P_{\ell}^{m}(\zeta) f_{m}^{q}(\varphi)-\left\{\mathbf{s}_{\ell, \text { in }}^{m / q}\right. \\
& \left.+\left[\frac{\mathbf{S}_{\ell}^{m / q}\left(\tau_{s}\right)}{P_{\ell}^{m}\left(\tau_{s}\right)}\right]\right\} \cdot \nabla u_{\ell, \mathrm{in}}^{m / q}\left(\tau_{s}, \zeta, \varphi\right)+\mathbf{s}_{\ell, \mathrm{ex}}^{m / q} \\
& \cdot \nabla u_{\ell, \mathrm{ex}}^{m / q}\left(\tau_{s}, \zeta, \varphi\right) .
\end{aligned}
$$

Bearing in mind, now, the two components of condition (77), then for $\kappa=2$ we are led to the relations

$$
\begin{aligned}
& \bar{f}_{\ell, 1}^{m / q, 2}=0, \\
& \bar{f}_{\ell, 2}^{m / q, 2}=-Q_{\ell}^{m}(\tau) P_{\ell}^{m}(\zeta) \sin \varphi f_{m}^{q}(\varphi), \\
& \bar{f}_{\ell, 3}^{m / q, 2}=Q_{\ell}^{m}(\tau) P_{\ell}^{m}(\zeta) \cos \varphi f_{m}^{q}(\varphi), \\
& \bar{g}_{\ell}^{m / q, 2}=\widehat{\boldsymbol{\tau}} \times\left[\left(\mathbf{s}_{\ell, \mathrm{in}}^{m / q}+\mathbf{I}_{\ell, \mathrm{ex}}^{m / q}\left(\tau_{s}, \zeta, \varphi ; \mathbf{r}_{0}\right)\right) P_{\ell}^{m}\left(\tau_{s}\right)\right. \\
&\left.+\mathbf{s}_{\ell, \mathrm{ex}}^{m / q} Q_{\ell}^{m}\left(\tau_{s}\right)+\mathbf{S}_{\ell}^{m / q}\left(\tau_{s}\right)\right] \cdot \widehat{\boldsymbol{\zeta}} P_{\ell}^{m}(\zeta) f_{m}^{q}(\varphi)=0
\end{aligned}
$$


with the $\kappa=3$ case being set by the relations

$$
\begin{aligned}
& \bar{f}_{\ell, 1}^{m / q, 3}=\tau_{s} Q_{\ell}^{m}\left(\tau_{s}\right) \sqrt{1-\zeta^{2}} P_{\ell}^{m}(\zeta) f_{m}^{q}(\varphi), \\
& \bar{f}_{\ell, 2}^{m / q, 3}=-\sqrt{\tau_{s}^{2}-1} Q_{\ell}^{m}\left(\tau_{s}\right) \zeta P_{\ell}^{m}(\zeta) \cos \varphi f_{m}^{q}(\varphi), \\
& \bar{f}_{\ell, 3}^{m / q, 3}=-\sqrt{\tau_{s}^{2}-1} Q_{\ell}^{m}\left(\tau_{s}\right) \zeta P_{\ell}^{m}(\zeta) \sin \varphi f_{m}^{q}(\varphi), \\
& \bar{g}_{\ell}^{m / q, 3}=\sqrt{\tau_{s}^{2}-\zeta^{2}} \widehat{\boldsymbol{\tau}} \\
& \times\left[\left(\mathbf{s}_{\ell, \mathrm{in}}^{m / q}+\mathbf{I}_{\ell, \mathrm{ex}}^{m / q}\left(\tau_{s}, \zeta, \varphi ; \mathbf{r}_{0}\right)\right) P_{\ell}^{m}\left(\tau_{s}\right)\right. \\
& \left.+\mathbf{s}_{\ell, \mathrm{ex}}^{m / q} Q_{\ell}^{m}\left(\tau_{s}\right)+\mathbf{s}_{\ell}^{m / q}\left(\tau_{s}\right)\right] \cdot \widehat{\boldsymbol{\varphi}} P_{\ell}^{m}(\zeta) f_{m}^{q}(\varphi) .
\end{aligned}
$$

Expressions (103)-(105) act for $\tau_{s} \neq \tau_{0}, \zeta \in[-1,1], \varphi \in$ $[0,2 \pi)$, and $\mathbf{r}_{0}=\left(\tau_{0}, \zeta_{0}, \varphi_{0}\right)$, where the vectors $\widehat{\boldsymbol{\tau}} \equiv \widehat{\boldsymbol{\tau}}\left(\tau_{s}, \zeta, \varphi\right)$, $\widehat{\boldsymbol{\zeta}} \equiv \widehat{\zeta}\left(\tau_{s}, \zeta, \varphi\right)$, and $\widehat{\boldsymbol{\varphi}} \equiv \widehat{\boldsymbol{\varphi}}(\varphi)$ are with (36)-(38), the dipole source $\mathbf{m}$ is given in $(2), \nabla u_{\ell, \text { in }}^{m / q}\left(\tau_{s}, \zeta, \varphi\right)$ and $\nabla u_{\ell, \text { ex }}^{m / q}\left(\tau_{s}, \zeta, \varphi\right)$ for $\ell \geq 0, m=0,1,2, \ldots, \ell$, and $q=e, o$ assume (48) and (49), respectively, derivations are with respect to the argument, and notations (94), (95), (98), and (101) have been implied. Those are expanded through the orthonormal basis $P_{\ell^{\prime}}^{m^{\prime}} f_{m^{\prime}}^{q^{\prime}}$ for $\ell^{\prime} \geq 0, m^{\prime}=0,1,2, \ldots, \ell^{\prime}$, and $q^{\prime}=e$, o so that

$$
\begin{array}{r}
\bar{f}_{\ell, j}^{m / q, \kappa}\left(\tau_{s}, \zeta, \varphi\right) \\
=\sum_{\ell^{\prime}=0}^{+\infty} \sum_{m^{\prime}=0}^{\ell^{\prime}} \sum_{q^{\prime}=e, o} \bar{\lambda}_{\left(\ell, \ell^{\prime}\right), j}^{\left(m, m^{\prime}\right) /\left(q, q^{\prime}\right), \kappa}\left(\tau_{s}\right) P_{\ell^{\prime}}^{m^{\prime}}(\zeta) f_{m^{\prime}}^{q^{\prime}}(\varphi) \\
\text { with } \kappa, j=1,2,3,
\end{array}
$$

$$
\begin{aligned}
& \bar{g}_{\ell}^{m / q, \kappa}\left(\tau_{s}, \zeta, \varphi ; \mathbf{r}_{0}\right) \\
& =\sum_{\ell^{\prime}=0}^{+\infty} \sum_{m^{\prime}=0}^{\ell^{\prime}} \sum_{q^{\prime}=e, o} \bar{\mu}_{\left(\ell, \ell^{\prime}\right)}^{\left(m, m^{\prime}\right) /\left(q, q^{\prime}\right), \kappa}\left(\tau_{s} ; \mathbf{r}_{0}\right) P_{\ell^{\prime}}^{m^{\prime}}(\zeta) f_{m^{\prime}}^{q^{\prime}}(\varphi)
\end{aligned}
$$$$
\text { with } \kappa=1,2,3
$$

as for $\ell \geq 0, m=0,1,2, \ldots, \ell$, and $q=e, o$, whereas projection in view of (44) and (45) reveals that

$$
\begin{gathered}
\bar{\lambda}_{\left(\ell, \ell^{\prime}\right), j}^{\left(m, m^{\prime}\right) /\left(q, q^{\prime}\right), \kappa}\left(\tau_{s}\right)=\frac{2 \ell^{\prime}+1}{4 \pi} \frac{\left(\ell^{\prime}-m^{\prime}\right) !}{\left(\ell^{\prime}+m^{\prime}\right) !} \\
\cdot \varepsilon_{m^{\prime}} \int_{-1}^{+1} \int_{0}^{2 \pi} \bar{f}_{\ell, j}^{m / q, \kappa}\left(\tau_{s}, \zeta, \varphi\right) P_{\ell^{\prime}}^{m^{\prime}}(\zeta) \\
\cdot f_{m^{\prime}}^{q^{\prime}}(\varphi) d \varphi d \zeta, \\
\bar{\mu}_{\left(\ell, \ell^{\prime}\right)}^{\left(m, m^{\prime}\right) /\left(q, q^{\prime}\right), \kappa}\left(\tau_{s} ; \mathbf{r}_{0}\right)=\frac{2 \ell^{\prime}+1}{4 \pi} \frac{\left(\ell^{\prime}-m^{\prime}\right) !}{\left(\ell^{\prime}+m^{\prime}\right) !} \\
\cdot \varepsilon_{m^{\prime}} \int_{-1}^{+1} \int_{0}^{2 \pi} \bar{g}_{\ell}^{m / q, \kappa}\left(\tau_{s}, \zeta, \varphi ; \mathbf{r}_{0}\right) P_{\ell^{\prime}}^{m^{\prime}}(\zeta) \\
\cdot f_{m^{\prime}}^{q^{\prime}}(\varphi) d \varphi d \zeta
\end{gathered}
$$

for every $\left(\ell, \ell^{\prime}\right) \geq 0,\left(m, m^{\prime}\right)=0,1,2, \ldots,\left(\ell, \ell^{\prime}\right)$, and $\left(q, q^{\prime}\right)=$ $e, o$ with $\kappa, j=1,2,3$. Including (106) and (116) with (107) and (108) within (102), we end up with

$$
\sum_{\ell=0}^{+\infty} \sum_{m=0}^{\ell} \sum_{q=e, o}\left[\sum_{j=1}^{3}\left(\bar{\lambda}_{\left(\ell, \ell^{\prime}\right), j}^{\left(m, m^{\prime}\right) /\left(q, q^{\prime}\right), \kappa}\left(\tau_{s}\right) d_{\ell, j}^{m / q}\right)-\bar{\mu}_{\left(\ell, \ell^{\prime}\right)}^{\left(m, m^{\prime}\right) /\left(q, q^{\prime}\right), \kappa}\left(\tau_{s} ; \mathbf{r}_{0}\right)\right]=0 \quad \text { for } \mathbf{r}_{0}=\left(\tau_{0}, \zeta_{0}, \varphi_{0}\right)
$$

while $\kappa=1,2,3$, as well $\ell^{\prime} \geq 0, m^{\prime}=0,1,2, \ldots, \ell^{\prime}$, and $q^{\prime}=$ $e, o$. In matrix form, (109) reads

$$
\begin{aligned}
\overline{\mathbb{A}}_{L}\left(\tau_{s}\right) \overline{\mathbf{x}}_{L} & =\overline{\mathbf{z}}_{L}\left(\tau_{s} ; \mathbf{r}_{0}\right) \Longrightarrow \\
\overline{\mathbf{x}}_{L} & =\overline{\mathbb{A}}_{L}^{-1}\left(\tau_{s}\right) \overline{\mathbf{z}}_{L}\left(\tau_{s} ; \mathbf{r}_{0}\right)
\end{aligned}
$$

$$
\text { for } \mathbf{r}_{0}=\left(\tau_{0}, \zeta_{0}, \varphi_{0}\right),
$$

which are linear algebraic systems, where, imposing a fictitious upper limit $\ell=\ell^{\prime}=0,1,2, \ldots, L$, then for any $m=$ $m^{\prime}=0,1,2, \ldots, \ell$ and $q=q^{\prime}=e, o$, as well as $\kappa, j=1,2,3$, we have

$$
\overline{\mathbb{A}}_{L}\left(\tau_{s}\right)=\left[\begin{array}{ccc}
\ddots & \vdots & \cdot \\
\cdots & \bar{\lambda}_{(\ell, \ell), j}^{(m, m) /(q, q), \kappa}\left(\tau_{s}\right) & \cdots \\
. & \vdots & \ddots
\end{array}\right],
$$

$$
\begin{aligned}
\overline{\mathbf{x}}_{L}= & {\left[\begin{array}{c}
\vdots \\
d_{\ell, j}^{m / q} \\
\vdots
\end{array}\right], } \\
\overline{\mathbf{z}}_{L}\left(\tau_{s} ; \mathbf{r}_{0}\right)= & {\left[\begin{array}{c}
\vdots \\
\bar{\mu}_{(\ell, \ell), j}^{(m, m) /(q, q), \kappa}\left(\tau_{s} ; \mathbf{r}_{0}\right) \\
\vdots
\end{array}\right], }
\end{aligned}
$$

which stand for the $3(L+1) \times m \times q$ squared-type invertible matrix of the coefficients of the unknowns, the vector of the unknown coefficients, and the vector of the known constants, respectively, of this particular problem. A standard cut-off method is applied to (110) in order to decide the truncation 
amplitude $L$, which supplies with an acceptable accuracy of a possible numerical implementation. Afterwards, the coefficients $\mathbf{d}_{\ell, \text { ex }}^{m / q}=d_{\ell, 1}^{m / q} \widehat{\mathbf{x}}_{1}+d_{\ell, 2}^{m / q} \widehat{\mathbf{x}}_{2}+d_{\ell, 3}^{m / q} \widehat{\mathbf{x}}_{3}$ are determined for $\ell=0,1,2, \ldots, L, m=0,1,2, \ldots, \ell$, and $q=e, o$, while the scattered electric field, comprising this situation, is built up from (86), encountering (89) and (99), that is,

$$
\begin{aligned}
& \mathbf{E}_{3}^{s}(\mathbf{r}) \\
& \quad=\sum_{\ell=0}^{+\infty} \sum_{m=0}^{\ell} \sum_{q=e, o}\left[\left(\mathbf{d}_{\ell, \mathrm{ex}}^{m / q}+\mathbf{s}_{\ell, \mathrm{ex}}^{m / q}\right) Q_{\ell}^{m}(\tau)+\mathbf{s}_{\ell, \mathrm{in}}^{m / q} P_{\ell}^{m}(\tau)+\mathbf{s}_{\ell}^{m / q}(\tau)\right] \\
& \quad \cdot P_{\ell}^{m}(\zeta) f_{m}^{q}(\varphi) \quad \text { for } \mathbf{r} \in \Omega,
\end{aligned}
$$

being established by the prolate spheroidal configuration of $\mathbf{d}_{\ell, \mathrm{ex}}^{m / q}$ in (90) for $\ell=0,1,2, \ldots, L, m=0,1,2, \ldots, \ell$, and $q=e, o$, once notations (94), (95), and (98) are considered.

3.4. Recapitulation: The Oblate Spheroid and Limiting Cases. Summarizing all the above analysis, we have retrieved in a closed-type analytical fashion the surviving low-frequency terms of the scattered magnetic $\mathbf{H}_{0}^{s}, \mathbf{H}_{2}^{s}, \mathbf{H}_{3}^{s}$ and electric $\mathbf{E}_{1}^{s}, \mathbf{E}_{3}^{s}$ fields under consideration in the prolate spheroidal domain of interest $\Omega$ from well-posed boundary value problems, while $\mathbf{H}_{1}^{s}=\mathbf{E}_{0}^{s}=\mathbf{E}_{2}^{s}=\mathbf{0}$. Collecting the associated terms (58), (76), (83) and (85), (112), respectively, we may substitute them into (21) and (22), so as to obtain the electromagnetic fields in a compact analytical form as infinite power series expansions, being ready for further possible numerical implementation.

Recapitulating and for reasons of completeness, we mention that the corresponding results of the oblate spheroidal geometry are recovered through the simple transformation [12]

$$
\tau \longrightarrow i \lambda, \quad c \longrightarrow-i \bar{c}
$$

where $0 \leq \lambda \equiv \sinh \eta<+\infty$ and $\bar{c}>0$ are the new characteristic variables of the system. On the other hand, the asymptotic case of the needle of length $a_{1}$ can be reached by a prolate spheroid when $0<a_{3}=a_{2} \ll a_{1}<+\infty$, while, for the case where $0<a_{3} \ll a_{2}=a_{1}<+\infty$, the oblate spheroid takes the shape of a circular disk of radius $a_{1}=a_{2}$.

\section{Analytical and Numerical Validation}

The spheroidal geometry yields a reliable and quite representative coordinate system when we have to deal with scattering wave problems, where the field is perturbed by a perfectly conducting body. However, this is true only when the lowfrequency approximation is implied, since we are able to deal with boundary value problems of Laplace's equation, which is fully separable in this geometry. Hence, problems similar to our case adopt this fitting system of coordinates to obtain analytical or semianalytical results for the corresponding fields.

Nevertheless, it is not always easy and feasible to pursue fully analytical solutions in closed forms without computational error, or the obtained closed formulae do not have the required accuracy, even in the low-frequency realm. Indeed, even though the scattered electromagnetic fields have been calculated for $n=0,1,2,3$ (higher order terms are not of substantial interest) in a closed analytical form of infinite series in terms of the spheroidal harmonic eigenexpansions, they are not given in fully compact fashion. This is due to the fact that the constant coefficients in some circumstances are evaluated up to a certain order of accuracy via cutoff techniques. There, accuracy is controlled by the sum of terms taken in each case, which inherits the semianalytical character to our method. To this end, we need validation methods, both analytical and numerical, in order to crosscheck the credibility of our results, those being the analytical reduction to the sphere-body case and the implementation of the obtained formulae.

4.1. Reduction to the Spherical Case. The prolate spheroidal geometry degenerates to the spherical one [12] when $c \rightarrow$ $0^{+}$. For the corresponding analytical reduction, the limiting process involves an appropriate combination of the semifocal distance with the coordinate variable $\tau>1$, such as

$$
\lim _{c \rightarrow 0^{+}} c \tau=r, \quad \lim _{c \rightarrow 0^{+}} \frac{1}{2 c} \ln \frac{\tau+1}{\tau-1}=\frac{1}{r}, \quad \text { where } r \equiv\|\mathbf{r}\|=c \sqrt{\tau^{2}+\zeta^{2}-1} \text { for }|\zeta| \leq 1
$$

where $r \geq 0$ is the radial component of the spherical coordinate system

$$
\begin{aligned}
& x_{1}=r \zeta, \\
& x_{2}=r \sqrt{1-\zeta^{2}} \cos \varphi, \\
& x_{3}=r \sqrt{1-\zeta^{2}} \sin \varphi, \\
& \quad \text { for } r \geq 0,|\zeta| \leq 1, \varphi \in[0,2 \pi),
\end{aligned}
$$

which is readily recovered from (33), pointing out that the other two radial variables remain unaltered, because they coincide in both systems. Obviously, if we multiply nominator and dominator of (36) and (37) by $c>0$ and use (114), we obtain the spherical radial unit normal vector

$$
\begin{aligned}
\lim _{c \rightarrow 0^{+}} \widehat{\boldsymbol{\tau}}(\tau, \zeta, \varphi)= & \widehat{\mathbf{r}}(\zeta, \varphi) \\
= & \zeta \widehat{\mathbf{x}}_{1}+\sqrt{1-\zeta^{2}} \cos \varphi \widehat{\mathbf{x}}_{2} \\
& +\sqrt{1-\zeta^{2}} \sin \varphi \widehat{\mathbf{x}}_{3} \equiv \frac{\mathbf{r}}{r}
\end{aligned}
$$

with $r \geq 0$, 
where $|\zeta| \leq 1$ and $\varphi \in[0,2 \pi)$, while the remaining vectors assume similar spherical form.

The corresponding reduction of formulae (58), (76), (83) and (85), (112) for the electromagnetic fields is mainly based on a proper limiting procedure, which is a consequence of the definition of the associated Legendre functions of the first and the second kind $[14,15]$, revealing that

$$
\begin{aligned}
& \lim _{c \rightarrow 0^{+}} c^{\ell} P_{\ell}^{m}(\tau)=p_{\ell} \frac{\ell !}{(\ell-m) !} r^{\ell}, \\
& \lim _{c \rightarrow 0^{+}} c^{-(\ell+1)} Q_{\ell}^{m}(\tau)=q_{\ell}(-1)^{m} \frac{(\ell+m) !}{\ell !} r^{-(\ell+1)}, \\
& \quad \text { for } r \geq 0,
\end{aligned}
$$

upon usage of (114), where for any value of $\ell \geq 0$ it is

$$
\begin{aligned}
& p_{\ell}=\frac{(2 \ell) !}{2^{\ell}(\ell !)^{2}}, \\
& q_{\ell}=\frac{1}{2^{\ell}} \sum_{k=0}^{[\ell / 2]} \frac{(-1)^{\kappa}(2 \ell-2 k) !}{k !(\ell-k) !(\ell-2 k) !(2 \ell-2 k+1)}, \\
& \text { with }(2 \ell+1) p_{\ell} q_{\ell}=1 .
\end{aligned}
$$

The degeneration of the aforementioned low-frequency electric and magnetic fields, in order to recover the case of a spherical metallic body of radius $c \tau_{s} \rightarrow r_{s}$, embedded within a lossless medium, is then a straightforward sequence of steps, since any kind of indeterminacies is absent.

4.2. Numerical Implementation. We adopt the appropriate prolate spheroidal geometry to describe a perfectly conducting $\left(\sigma_{\text {body }} \rightarrow+\infty\right)$ spheroidal body of major and minor axis $a_{1}=75 \mathrm{~m}$ and $a_{2}=a_{3}=50 \mathrm{~m}$, respectively; therefore, taking the semifocal distance as $c=25 \mathrm{~m}$, the characteristic prolate spheroidal variable is $\tau_{s}=3$. We wish to implement the most frequently measurable field, which is the scattered magnetic field $\mathbf{H}^{s}$, given by expression (21), implying the low-frequency solutions (58), (76), and (83). The particular geometry we use to represent the metallic target, which is embedded in a homogeneous infinite space of permeability $\mu \cong \mu_{0}=$ $4 \pi \times 10^{-7} \mathrm{~F} / \mathrm{m}$ and permittivity $\varepsilon \cong \varepsilon_{0}=8.854 \times 10^{-12} \mathrm{~F} / \mathrm{m}$ (values of vacuum), is shown in Figure 1, whereas the axis of symmetry is comprised by the $x_{1}$-axis.

In order to provide understandable and symmetric diagrams so as to validate the achieved relations for the implicated fields, we choose a magnetic dipole source $\mathbf{m}=m_{1} \widehat{\mathbf{x}}_{1}$ of strength $m_{1}=4 \pi \times 10^{3} \mathrm{~A} \cdot \mathrm{m}^{2}$, which is located at $\mathbf{r}_{0} \equiv$ $\left(x_{10}, x_{20}, x_{30}\right)=(200,0,0) \mathrm{m}$ and illuminates the prolate spheroid at the low frequency of $\omega=2 \pi v$, where $v=50 \mathrm{~Hz}$, while the magnetic field is measured along a line parallel to the $x_{1}$-axis, set at $\left(x_{1}, x_{2}, x_{3}\right)=([-200,200], 200,0) \mathrm{m}$. We show numerical results by providing illustrations for the real and the imaginary parts of the complex approximated scattered magnetic field in units $[\mathrm{A} / \mathrm{m}]$, readily shown in the sequel within Figure 2.

Additionally, under the aim of providing numerical demonstrations for the limiting occasions of the prolate spheroid, we also plot the two interesting limits of the needle with length $a_{1}=75 \mathrm{~m} \gg a_{2}=a_{3}=5 \mathrm{~m}$ and the sphere of radius $a=50 \mathrm{~m}=a_{1}=a_{2}=a_{3}$. Furthermore, in order to compare all three cases, we insert in the same figure (Figure 2) both the needle and the sphere circumstances, along with the general case of a genuine prolate spheroidal object.

The series solution obtained for the evaluation of $\mathbf{H}^{s}$ converges relatively fast as $\ell \rightarrow+\infty$, while it must be pointed out that a few terms of the series expansions $\ell \cong 20$ are enough for determining the scattered magnetic field within a very good accuracy. Moreover, the number of the adequate terms needed to converge and obtain a specific accuracy depends upon the ratio of the major axes over one of the two minor axes of the prolate spheroid, which means an increase of $\ell$ as this ratio increases. Certainly, the number of the utilized terms is increased, a fact that it is partly justified by the necessity of the applied cut-off techniques. Further discussion involves the numerical behavior of the analytical solution, presented in Figure 2. Obviously, the $x_{3}$ components are absent, since we have chosen our observation line at $x_{3}=0$. As expected and in accordance with the position of the dipole, the results are symmetric with respect to the line $x_{1}=0$. On the other hand, all the three situations behave similarly, while in terms of magnitudes there is no significant change between the prolate spheroidal and the spherical case, which is not the fact for the case of a needle, meaning that, for extreme circumstances, there is a slight difference in the final result.

\section{Conclusions and Discussion}

An analytical method to the electromagnetic low-frequency scattering problem for a perfectly electrically conducting spheroidal body in a conductive surrounding, which is illuminated by a magnetic dipole with arbitrary close to the object location and orientation, is developed. The general methodology for the identification of the metal spheroid is presented, based on rigorous, yet versatile, mathematical tools. Its highly conducting morphology is taken into account via impenetrable conditions, while the limiting behavior of the fields at infinity is readily secured. This radiation approximation of a vanishing field at sufficiently long distance from the source creates an error of minor significance. Upon definition of the medium's wave number, the threedimensional fields assumed expansions at low frequencies, where a few terms are needed to provide an accurate description of the phenomenon. The spheroidal coordinate system was best fitted to the physical configuration of the problem and therein the 3D solution has been obtained in terms of handy spheroidal eigenfunctions. The commensurable spherical occasion is recovered via a careful limiting procedure, which demonstrated our cumbersome analysis at hand.

Suchlike analytical solutions and formulae attaining closed-type forms have quite important advantages compared with the pure numerical methods. Indeed, the validity of numerical solutions can be verified by analytical or semianalytical techniques. On the other hand, bearing in mind that very important physical laws can be derived from analytical 

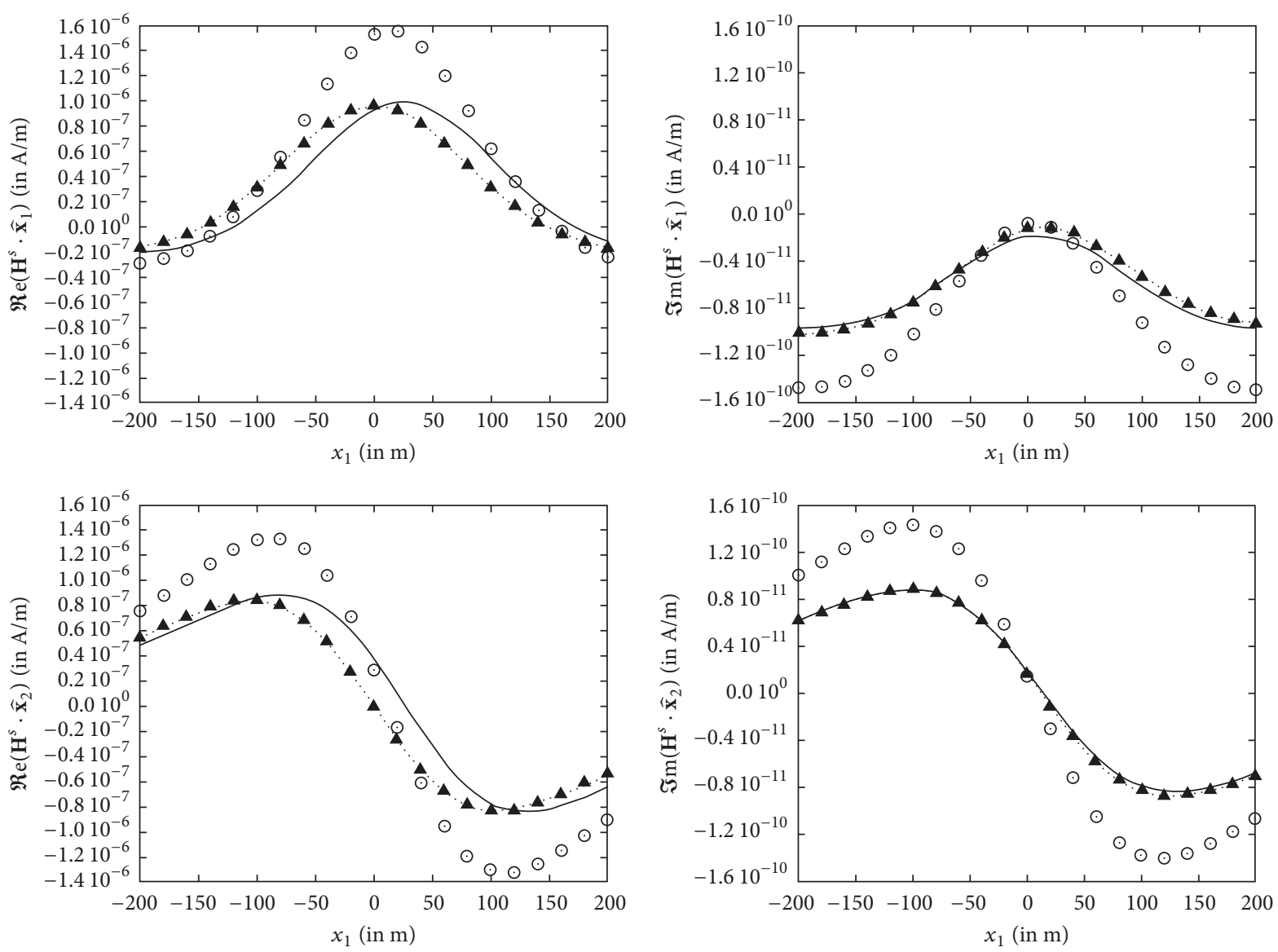

FIGURE 2: The $x_{1}$-components and $x_{2}$-components of the real and the imaginary parts of the approximated low-frequency scattered magnetic field $\mathbf{H}^{s}$, obtained on the $x_{1}$-axis for a prolate spheroid $(\cdots \boldsymbol{\Lambda} \cdots)$, a needle $(\odot)$, and a sphere $(-)$. The $x_{3}$-components are all vanishing.

methods, we can understand the necessity of tackling with a confident mathematical basis before incorporating with an algorithmic procedure. Therefore, even nowadays, there is always room for such kind of methods that coexist with pure numerical codes and aim to the solution of boundary value problems in physical applications of major importance.

Mathematical and computational work is currently in progress and involves research into several directions, such as the introduction of more complicated geometries for the metallic target or the accomplishment of actually difficult inversion algorithms, taking profit from the proposed framework.

\section{Conflicts of Interest}

The author declares that they have no conflicts of interest.

\section{References}

[1] J. A. Stratton, Electromagnetic Theory, John Wiley \& Sons, Inc., Hoboken, NJ, USA, 2015.

[2] G. Dassios and R. Kleinman, Low Frequency Scattering, Oxford University Press, Oxford, UK, 2000.
[3] H. Ammari and H. Kang, Polarization and Moment Tensors: with Applications to Inverse Problems and Effective Medium Theory, vol. 162 of Applied Mathematical Sciences Series, Springer, New York, NY, USA, 2007.

[4] H. Ammari, J. Garnier, W. Jing et al., Mathematical and Statistical Methods for Multistatic Imaging, vol. 2098 of Lecture Notes in Mathematics, Springer, 2013.

[5] P. Vafeas, G. Perrusson, and D. Lesselier, "Low-frequency solution for a perfectly conducting sphere in a conductive medium with dipolar excitation," Progress in Electromagnetics Research, vol. 49, pp. 87-111, 2004.

[6] G. Mie, "Beiträge zur Optik trüber Medien, speziell kolloidaler Metallösungen," Annalen der Physik, vol. 330, no. 3, pp. 377-445, 1908.

[7] P. Vafeas, G. Perrusson, and D. Lesselier, "Low-frequency scattering from perfectly conducting spheroidal bodies in a conductive medium with magnetic dipole excitation," International Journal of Engineering Science, vol. 47, no. 3, pp. 372-390, 2009.

[8] G. Perrusson, P. Vafeas, and D. Lesselier, "Low-frequency dipolar excitation of a perfect ellipsoidal conductor," Quarterly of Applied Mathematics, vol. 68, no. 3, pp. 513-536, 2010.

[9] G. Perrusson, P. Vafeas, I. K. Chatjigeorgiou, and D. Lesselier, "Low-frequency on-site identification of a highly conductive body buried in Earth from a model ellipsoid," IMA Journal of Applied Mathematics, vol. 80, no. 4, pp. 963-980, 2015. 
[10] P. Vafeas, P. K. Papadopoulos, and D. Lesselier, "Electromagnetic low-frequency dipolar excitation of two metal spheres in a conductive medium," Journal of Applied Mathematics, vol. 2012, Article ID 628261, 37 pages, 2012.

[11] P. Vafeas, P. K. Papadopoulos, P.-P. Ding, and D. Lesselier, "Mathematical and numerical analysis of low-frequency scattering from a PEC ring torus in a conductive medium," Applied Mathematical Modelling: Simulation and Computation for Engineering and Environmental Systems, vol. 40, no. 13-14, pp. 6477-6500, 2016.

[12] P. Moon and D. E. Spencer, Field Theory Handbook, Springer, Berlin, Germany, Second edition, 1988.

[13] P. M. Morse and H. Feshbach, Methods of Theoretical Physics, McGraw-Hill, New York, NY, USA, 1953.

[14] E. W. Hobson, The Theory of Spherical and Ellipsoidal Harmonics, Chelsea Publishing Company, New York, NY, USA, 1965.

[15] "Digital Library of Mathematical Functions (dlmf.nist.gov)".

[16] P. Vafeas, D. Lesselier, and F. Kariotou, "Estimates for the low-frequency electromagnetic fields scattered by two adjacent metal spheres in a lossless medium," Mathematical Methods in the Applied Sciences, vol. 38, no. 17, pp. 4210-4237, 2015.

[17] P. Vafeas, "Low-frequency electromagnetic scattering by a metal torus in a lossless medium with magnetic dipolar illumination," Mathematical Methods in the Applied Sciences, vol. 39, no. 14, pp. 4268-4292, 2016. 


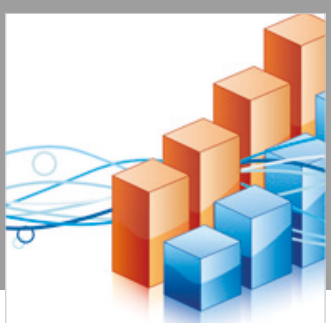

Advances in

Operations Research

\section{-n-m}
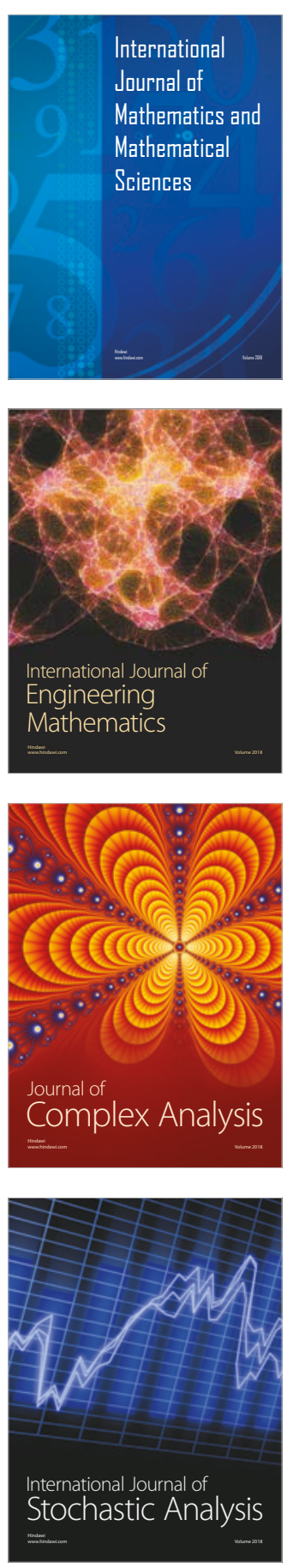
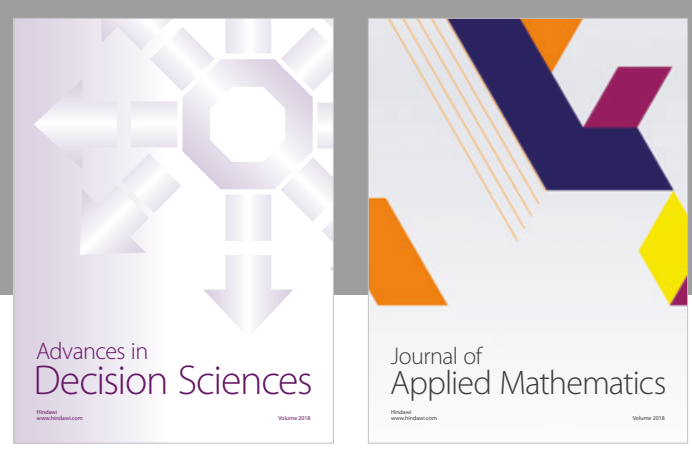

Journal of

Applied Mathematics
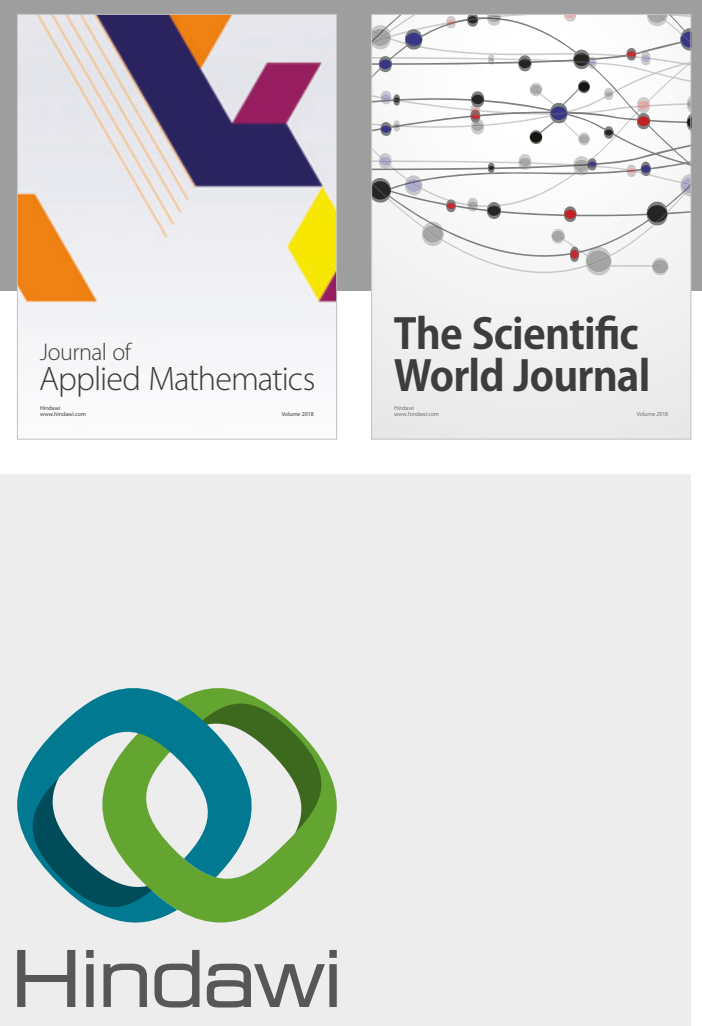

Submit your manuscripts at

www.hindawi.com

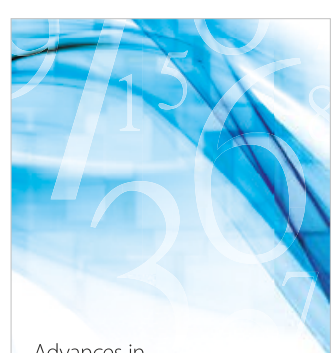

Advances in
Numerical Analysis
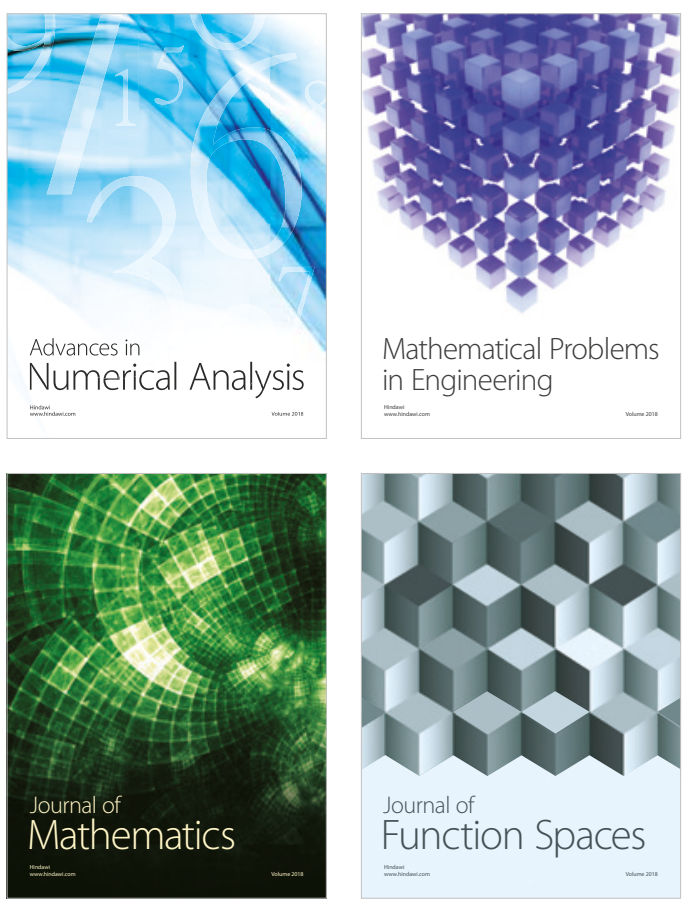

Mathematical Problems in Engineering

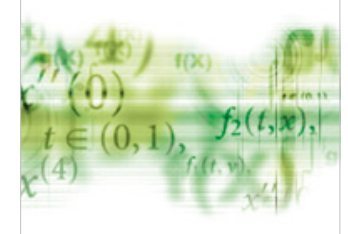

International Journal of

Differential Equations

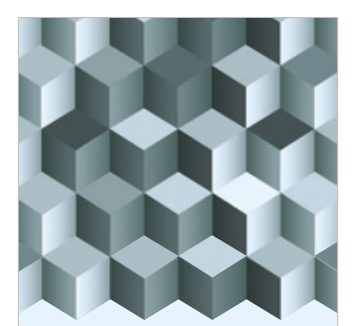

Journal of

Function Spaces

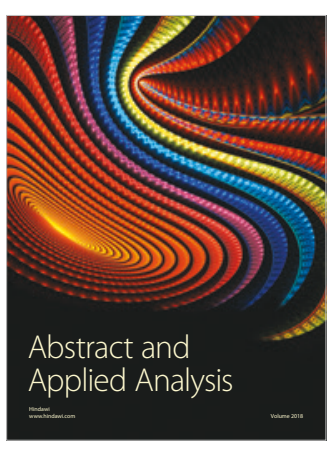

The Scientific

World Journal

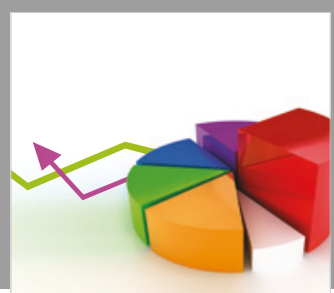

Journal of

Probability and Statistics
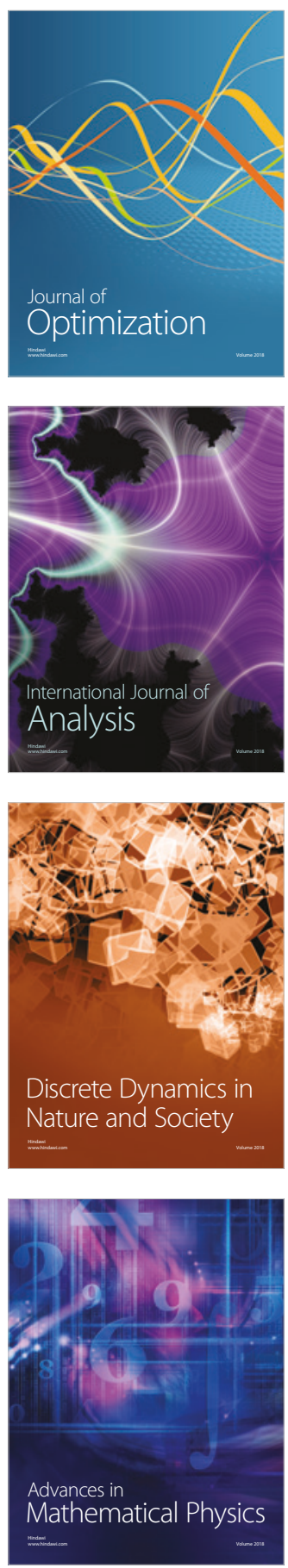